\title{
Activation of the membrane-bound Nrf1 transcription factor by USP19, a tail-anchored ubiquitin-specific protease in the endoplasmic reticulum
}

\author{
Shaofan Hu${ }^{1}$, Yuancai Xiang ${ }^{1,2}$, Lu Qiu ${ }^{1,3}$, Meng Wang ${ }^{1}$, and Yiguo Zhang ${ }^{1, *}$ \\ ${ }^{1}$ The Laboratory of Cell Biochemistry and Topogenetic Regulation, College of Bioengineering and Faculty of Medical Sciences, \\ Chongqing University, No. 174 Shazheng Street, Shapingba District, Chongqing 400044, China \\ ${ }^{2}$ Department of Biochemistry and Molecular Biology, College of Basic Medical Sciences, Southwest Medical University, Sichuan, \\ 646000 , China \\ ${ }^{3}$ School of Life Sciences, Zhengzhou University, No. 100 Kexue Avenue, Zhengzhou 450001, Henan, China \\ * Correspondence: yiguozhang@cqu.edu.cn, or eaglezhang64@gmail.com
}

\section{Abstract}

The membrane-bound transcription factor Nrf1 (i.e., encoded by Nfe2/1) is activated by sensing glucose deprivation, cholesterol excess, proteasomal inhibition and oxidative stress, and then mediates distinct signaling responses in order to maintain cellular homeostasis. Here, we found that Nrf1 stability and transactivity are enhanced by USP19, a tail-anchored ubiquitin-specific protease in the endoplasmic reticulum (ER). Further experiments revealed that USP19 directly interacts with Nrf1 in proximity to the ER and acts as a deubiquitinating enzyme to remove ubiquitin moieties from this protein and hence circumvent potential proteasomal degradation. Such USP19-mediated effect takes place only after Nrf1 is retrotranslocated by p97 out of ER membranes. Conversely, knockout of USP19 causes significant decreases in Nrf1 abundance and its active isoform entering the nucleus, resulting in down-regulation of its target proteasomal subunits. This led to a modest reduction of USP19-1--derived tumor growth in xenograft mice, when compared with wild-type controls. Altogether, these demonstrate that USP19 serves as a novel mechanistic modulator of Nrf1, but not Nrf2. In turn, our additional evidence has also unraveled that transcriptional expression of endogenous USP19 and its promoter-driven reporter genes is regulated by Nrf2, as well by Nrf1, at distinct layers within a complex hierarchical regulatory network.

Key words: Nrf1, Nrf2, USP19, proteasome, ubiquitination, deubiquitination, endoplasmic reticulum

\section{Introduction}

Nrf1 was identified as an ER membrane-bound transcription factor (Zhang et al., 2006; Zhang et al., 2007), that belongs to the Cap'n'Collar (CNC) basic-region leucine zipper (bZIP) family (Sykiotis and Bohmann, 2010; Zhang and Xiang, 2016). This family comprises nuclear factor-erythroid 2 (NF-E2) p45 subunit and related Nrf1, Nrf2, Nrf3, Bach1 and Bach2 in vertebrates, the Caenorhabditis elegans protein Skn-1 and the founding Drosophila melanogaster Cnc, also including another early-evolved subgroup of Nach (Zhu et al., 2018). They are essential for transcriptional regulation of distinct subsets of critical cognate genes, that are responsible for homeostasis, development, health and disease (Bugno et al., 2015; Gegotek and Skrzydlewska, 2015; Lynn and Curran, 2015; Yamamoto et al., 2018). Such target genes contain antioxidant response elements (AREs, 5'-TGAC/GnnnGC-3') and/or other cis-regulatory homologues (e.g., AP1-binding site, 5'-TGAC/GTCA-3') in their promoter regions. Of note, Nrf1 and Nrf2 are two principal CNC-bZIP factors in mammals, each of which directly binds ARE-driven genes through distinct functional heterodimers with a partner of small Maf (sMaf) or other bZIP proteins (e.g., AP1 and ATF4). In fact, gene-targeting experiments have also revealed that Nrf1 and Nrf2 fulfill distinct biological functions through regulating different subsets of ARE-driven genes (Hayes et al., 2020; Zhang and Xiang, 2016). Such distinctions between both CNC-bZIP factors are dictated by their different tempo-spatial processing mechanisms.

Accumulating evidence has also demonstrated that Nrf1 acts as an important ER sensor for intracellular redox, glucose, protein and lipid changes (Steffen et al., 2010; Widenmaier et al., 2017; Zhu et al., 2019). In response to those biological cues, the ER-resident Nrf1 is topologically dislocated across membranes into extra-ER subcellular compartments, where this protein is subjected to its selective post-translational processing (e.g., deglycosylation, 
bioRxiv preprint doi: https://doi.org/10.1101/2020.10.05.326363; this version posted October 6, 2020. The copyright holder for this preprint (which was not certified by peer review) is the author/funder, who has granted bioRxiv a license to display the preprint in perpetuity. It is made available under aCC-BY-NC-ND 4.0 International license.

deubiquitination, and juxtamembrane proteolysis) to yield a mature CNC-bZIP factor before transactivating cognate genes (e.g. those encoding proteasomal subunits, antioxidant proteins and detoxifying enzymes). By contrast, the water-soluble Nrf2 is segregated primarily in the cytoplasm by Keap1, an adaptor subunit of the Cullin 3-based E3 ubiquitin ligase that targets this CNC-bZIP protein to ubiquitin-mediated proteasomal degradation (Yamamoto et al., 2018). Whilst Keap1 acts as a key sensor for oxidative and electrophilic stress, it allows for dissociation from Nrf2, so that the CNC-bZIP factor is released and then translocated into the nucleus, where it transactivates target genes (involved in antioxidant, detoxification and cytoprotective adaptation).

Such distinct tempo-spatial processing mechanisms between Nrf1 and Nrf2 are attributable to their different structural domains. Of note, extra NTD (N-terminal domain) and NST (asparagine/serine/threonine-rich) domains are present in Nrf1, but not in Nrf2 (Zhang and Xiang, 2016). Within NTD, the NHB1 signal peptide allows Nrf1 to be anchored in a proper topological fold within and around ER membranes, and then the NHB2-adjoining peptide is responsive to the topobiologically-regulated juxtamembrane proteolytic processing of this CNC-bZIP protein (Xiang et al., 2018a; Xiang et al., 2018b). Once Nrf1 is anchored in the ER, its NST domain is partitioned in the lumen, in which it is glycosylated to become an inactive glycoprotein and thus protected by membranes (Zhang et al., 2007). As required, some ER luminal-resident domains of Nrf1 are allowed for dynamic retro-translocation into extra-ER compartments, which is driven predominantly by p97/VCP (Sha and Goldberg, 2014). In the topovectorial process, Nrf1 is losing the protection by membranes, such that its deglycosylated protein enables to be processed to yield a mature CNC-bZIP factor or otherwise degraded by proteasomes (Hamazaki and Murata, 2020).

During dynamic dislocation of Nrf1 from the ER lumen, it is postulated to be ubiquitinated by the ER-associated E3 ubiquitin ligase Hrd1 (Steffen et al., 2010; Tsuchiya et al., 2011; Xiang et al., 2018b), which also triggers protein retro-translocation across membranes into the cytoplasm (Baldridge and Rapoport, 2016; Hwang et al., 2017). The ubiquitin-labeled Nrf1 can be recognized and degraded by proteasomes. However, the ubiquitination of Nrf1 may be reversible, like most regulatory modifications of other proteins (Ventii and Wilkinson, 2008). Such being the case, it is inferable that ubiquitinated Nrf1 enables to be targeted for deubiquitination by an ubiquitin-specific protease (UPS) or another deubiquitinating enzyme (DUB), so that this CNC-bZIP protein is circumvented from the ubiquitinmediated proteasomal degradation. Amongst these UPS/DUBs, USP15 was reported to enable deubiquitination of Keap1, so as to efficiently incorporate into the Keap1-Cul3-E3 ligase complex and thus enhance the enzymatic activity to increase Nrf2 degradation, but with a concomitant reduction in Nrf2-target gene expression profile (Villeneuve et al., 2013). Later, USP15 was also showed to activate Nrf1 in the nucleus by stabilizing this CNC-bZIP factor through their physical interaction and ensuing deubiquitination (Fukagai et al., 2016). Together, USP15 can negatively regulate Nrf2 through deubiquitination of Keap1, but directly activates Nrf1-mediated expression of a PSMA4-derived ARE-luciferase reporter and endogenous proteasomal activity. In turn, Nrf1 was demonstrated to transactivate expression of USP9x, USP14 and other USP/DUB genes, in addition to those encoding proteasomal subunits, p97 and its co-factors (Sha and Goldberg, 2014; Taniguchi et al., 2017). Nonetheless, it is unknown whether the membrane-bound Nrf1 is regulated by one of the ER-resident USP enzymes [i.e., USP19, USP30 or USP48, that were identified by(Hassink et al., 2009)].

Of note, USP19 has been showed to rescue the substrates of ER-associated degradation (ERAD) by removing their ubiquitin chain in unfolded protein response (UPR) (Hassink et al., 2009). In the meanwhile, USP19 also participates in the unconventional secretion to export misfolded cytosolic proteins (e.g., neurodegenerative disease-causing proteins) into extracellular space(Xu et al., 2018). In the protein-disposing mechanism, USP19 binds HSP70/HSC70 and also acts upstream of HSC70 and DNAJC5 (a membrane-associated chaperone in late endosomes and lysosomes). Thus, USP19 is involved in regulation of cell cycle progression, DNA damage repair, apoptosis and autophagy (Lei et al., 2019; Miao et al., 2020). Here, we found that USP19 interacts with Nrf1 to enhance this CNC-bZIP stability and its transcriptional activity. This is due to the observation that a half-life of Nrf1 is significantly prolonged by 
over-expression of USP19, but shortened in USP19-1- cells. Such effects of USP19 on Nrf1 are nearly completely prevented by mutating all six putative ubiquitination sites within NTD and AD1 of this CNC-bZIP protein. Loss of USP19 causes a reduction in Nrf1 abundances to enter the nucleus, along with reduced expression of its target proteasomal subunits. Consequently, a modest reduction of USP19-/--derived tumor growth was also observed in xenograft model mice. Overall, these findings demonstrate that USP19 serves as a novel modulator of Nrf1 to activate its target gene expression.

\section{Results}

USP19 enhances the protein abundance of Nrf1 and its transcriptional activity

The DUB/USP family members are distributed throughout distinct cellular locations to exert different functions (Clague et al., 2019; Coyne and Wing, 2016; Eletr and Wilkinson, 2014). Rather, it is worth mentioning that a subtle transmembrane (TM) structure exists in USP19, USP30 and USP48, but not USP14 or USP15, which enables each of the TM-containing proteases to be anchored within ER membranes (Hassink et al., 2009). Herein, five expression constructs for USP14, USP15, USP19, USP30 and USP48 were created (Fig. 1A), to examine the effect of potential deubiquitinating enzymes on Nrf1. As shown in Fig. 1B, co-transfection of each indicated USP with Nrf1 revealed no obvious changes in the full-length glycoprotein of Nrf1, but only co-expressing USP19 caused an extra conspicuous isoform to emerge (as marked by red star). This putative deubiquitinated isoform had a slightly less mass than the intact glycoprotein-A of Nrf1, but its molecular weight is much more than Nrf1 deglycoprotein-B.
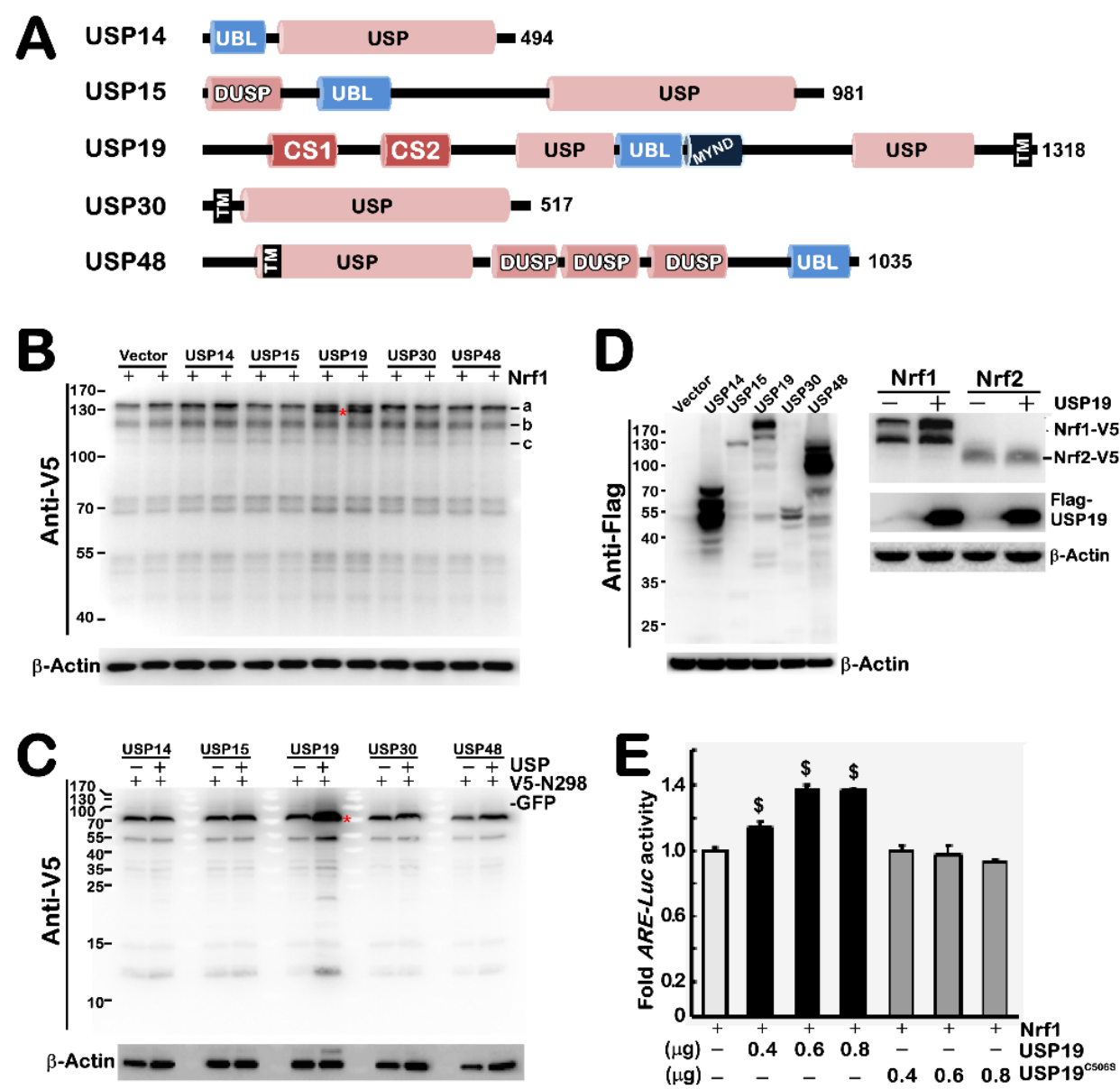

Figure 1. The deubiquitinating effect of USP19 on Nrf1, but not Nrf2.

(A) Schematic of five ubiquitin-specific proteases (USPs) with distinct domains. Their deubiquitinating enzymatic activity is dictated by their founding USP domain. Besides, specific DUSP (domain present in the USP family) can regulate the 
bioRxiv preprint doi: https://doi.org/10.1101/2020.10.05.326363; this version posted October 6, 2020. The copyright holder for this preprint (which was not certified by peer review) is the author/funder, who has granted bioRxiv a license to display the preprint in perpetuity. It is made available under aCC-BY-NC-ND 4.0 International license.

stability of or interact with HIF-1 $\alpha$, VHL or COP9-signalosome-associated cullin E3 ligase complexes, while CS (CHORD and SGT1) domain has a feature of cochaperone interacting with multiple protein complexes. Additional locations of ubiquitin-like (UBL), zinc-finger Myeloid-Nervy-DEAF1 (MYND) and transmembrane (TM) domains are also indicated.

(B, C) Expression constructs for Nrf1-V5 (B) or V5-N298-eGFP (C) plus one of deubiquitinating enzymes USP14, USP15, USP19, USP30 and USP48, were co-transfected into COS-1 cells, followed by Western blotting of the cell lysates with V5 antibody.

(D) Left panel shows protein expression of those indicated USPs in the above-transfected COS-1 cells. Right panel shows an effect of USP19 on Nrf1, but not Nrf2, in their co-expressing COS-1 cells.

(E) A dose-dependent effect of USP19 on Nrf1-mediated 6×ARE-Luc reporter activity in COS-1 cells, that had been co-transfected for $8 \mathrm{~h}$ with an expression construct for Nrf1, together with different amounts ( $\mu$ g of cDNAs) of USP19 or mutant USP19 ${ }^{\mathrm{C5065}}$, plus $6 \times A R E$-Luc or pRL-TK plasmids and then allowed for $24-\mathrm{h}$ recovery from transfection. The resulting data were calculated as fold changes (mean \pm SEM, $n=3 \times 3$ ) with significant increases $(\$, p<0.01)$, relative to the control value obtained from without USP19.

Further examination unraveled that the intact full-length chimeric protein V5-N298-GFP (in which N298 (i.e. the first 298 aa of Nrf1) was sandwiched between the N-terminal V5 tag and C-terminal GFP, as described (Xiang et al., 2018a)) of 80-kDa, as well as its several N-terminally-cleaved polypeptides of between 55-kDa and 12.5-kDa, were markedly increased by co-expression of USP19 (Fig. 1C). However, no similar effects were obtained from other four proteases examined. These imply that the proteolytic processing of N298 by USP19 occurs within the NTD and AD1 of Nrf1. Rather such effects of USP19 on Nrf1 appeared to be not exerted on Nrf2 (Fig. 1D, right panel). This distinction may be attributable to a few of structural domains (e.g., NTD) in Nrf1, but not in Nrf2, albeit both factors shared several conserved functional domains (Zhang and Xiang, 2016).

The transactivation activity of Nrf1 to mediate transcriptional expression of a 6xARE-driven luciferase reporter were incremented by USP19 in a dose-dependent manner (Fig. 1E). Such USP19-increased transactivity of Nrf1 was completely abolished by USP19 ${ }^{\text {C506s }}$ (a mutant of the enzymatic thiol-active site of USP19 at Cys ${ }^{506}$ into serine). This indicates that transactivation activity of Nrf1 is regulated by USP19 to remove its ubiquitin moieties.

USP19 is required for the stability of Nrf1 with a prolonged half-life

To determine the effects of USP19 on Nrf1 stability and processing, pulse-chase experiments were carried out by using distinct cell lines that were treated with $50 \mu \mathrm{g} / \mathrm{ml}$ of cycloheximide ( $\mathrm{CHX}$, to inhibit biosynthesis of nascent proteins). Western blotting of cell lysates showed that abundances of Nrf1 $\alpha$ and derivative isoforms were strikingly enhanced by USP19; this led to an extended course of $\mathrm{CHX}$-chased immunoblots before their disappearance by 4-h treatment, when compared to control experiments without this deubiquitinating protease (Figs. $2 A$ and S1A,B). The difference in the conversion between Nrf1 $\alpha$-derived isoforms was analyzed stoichiometrically (Fig. 2B). The stability of Nrf1 $\alpha$-derived glycoprotein (G), deglycoprotein (D) and processed proteins (P) (which were separated by distinct electrophoretic gels (Fig. S1A,B) was estimated by their half-lives, which were determined to be 0.40 (vs 0.28 ), 2.99 (vs 1.39), and 3.48 (vs 0.46) h after CHX treatment of COS-1 cells co-expressing USP19 (vs not), respectively (Fig. 2B). Conversely, knockout of USP19 from HepG2 cells (Fig.S2) resulted in obvious decreases in abundances of Nrf1 $\alpha$ derived proteins (Figs. 2C and S1C,D), along with their shortened half-lives in USP19-/- cells (as shown graphically in Fig. 2D), by comparison with wild-type controls.

Further examination of USP19's effects on endogenous Nrf1 stability revealed that distinct half-lives of its three major isoforms-A, B and C were slightly prolonged by over-expression of this protease (Figs. 2E and S1E), as shown graphically (Fig. 2F). However, half-lives of the endogenous Nrf1 $\alpha$-derived isoforms-A, B and C were substantially shortened by knockout of USP19-/- (Fig. 2G and S1F), which were determined to be $0.62,1.69,0.72 \mathrm{~h}$, respectively, 
bioRxiv preprint doi: https://doi.org/10.1101/2020.10.05.326363; this version posted October 6, 2020. The copyright holder for this preprint (which was not certified by peer review) is the author/funder, who has granted bioRxiv a license to display the preprint in perpetuity. It is made available under aCC-BY-NC-ND 4.0 International license.

(Fig. 2H), when compared with their equivalent controls in wild-type cells that were estimated by half-lives of 0.93 , 2.47, and $1.58 \mathrm{~h}$ after CHX treatment. Together, these demonstrate a requirement of USP19 for Nrf1 stability, albeit nuanced effects of endogenous and ectopic USP19 on Nrf1 $\alpha$-derived proteins.
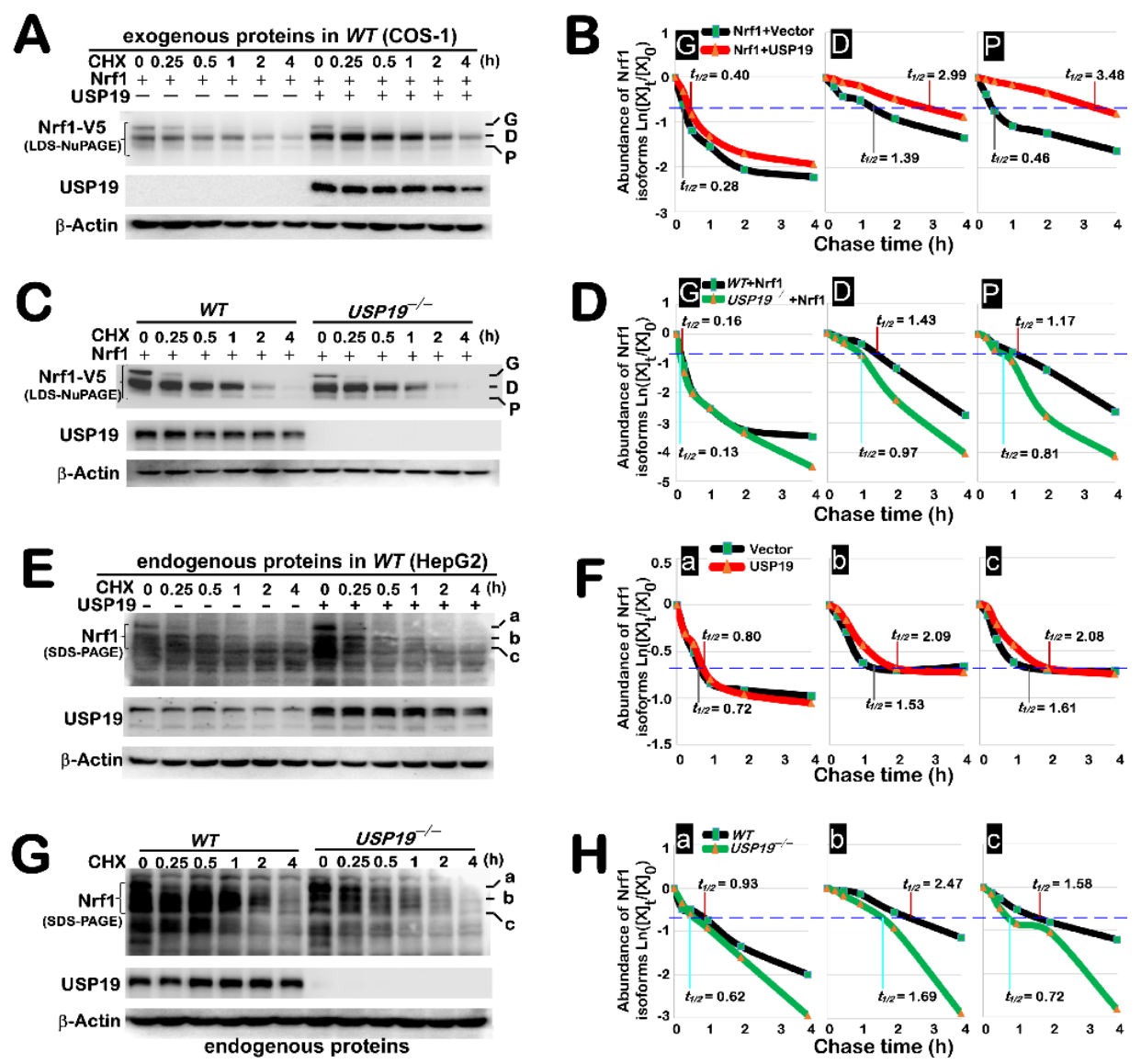

Figure 2. USP19 has an effect on the stability of Nrf1 isoforms with distinct half-lives.

(A, B) COS1 cells expressing Nrf1 alone or plus USP19 were treated with CHX for indicated lengths of time and then subjected to the pulse-chase analysis. The cell lysates were separated by 4-12\% LDS-NuPAGE gels in pH 7.7 running buffer, and visualized by Western blotting with antibodies against USP19 or V5 tag. Subsequently, the intensity of immunoblots representing Nrf1 isoforms was quantified by Quantity One, as shown graphically (B).

(C,D) Wild-type HepG2 and USP19-/- cell lines were allowed for ectopic expression of Nrf1, before being subjected to the pulse-chase experiments (C) and subsequent stoichiometrical analysis of Nrf1 isoforms (D).

(E, F) HepG2 cells expressing USP19 or not were subjected to CHX pulse-chase analysis of endogenous Nrf1 isoforms. The lyastes were separated by $8 \%$ SDS-PAGE gels in $\mathrm{pH} 8.3$ running buffer, and visualized by immunoblotting with antibodies against Nrf1 or USP19 (E). The stoichiometrical results of Nrf1 isoforms are shown graphically (F).

$(\mathrm{G}, \mathrm{H}) \mathrm{CHX}$-treated HepG2 (WT) and USP19-- cell lines were employed for time-course analysis of endogenous Nrf1 isoforms $(G)$, with distinct stability as shown graphically $(H)$.

Deubiquitination of Nrf1 by its interactor USP19 in close proximity to the ER

Distinct immunofluorescent images of COS-1 cells that had been co-transfected with two expression constructs for USP19 plus Nrf1 or Nrf1 ${ }^{\Delta 2-36}$ were visualized by confocal microscopy (Fig. 3A). Red fluorescent signals of USP19 are dominantly in the cytoplasm, and superimposed with those green signals of ER-resident Nrf1, albeit another fraction of this CNC-bZIP signals were distributed in the nucleus. By contrast, Nrf1 ${ }^{\Delta 2-36}$ gave predominant signals in the nucleus, where it cannot have fully engaged with the cytoplasmic USP19. 
bioRxiv preprint doi: https://doi.org/10.1101/2020.10.05.326363; this version posted October 6, 2020. The copyright holder for this preprint (which was not certified by peer review) is the author/funder, who has granted bioRxiv a license to display the preprint in perpetuity. It is made available under aCC-BY-NC-ND 4.0 International license.

Co-immunoprecipitation (IP) of the above cell lysates with V5 antibody showed a direct interaction of Nrf1-V5 with flagged USP19 (Fig. 3B, upper right lanes). Further anti-V5 Co-IP assays of the other cell lysates, that had been co-transfected with expression constructs for Nrf1 and HA-UB together with USP19 or not, revealed that abundance of ubiquitin-conjugated Nrf1-V5 was substantially reduced by this deubiquitinating protease (Fig. 3C, left panel).

Further experiments determined that USP19-deubiquitinated Nrf1 isoform (marked by red star) was abolished by USP19 ${ }^{\mathrm{C506S}}$ or USP19 ${ }^{\triangle T M}$ (lacking the C-terminal transmembrane (TM) region of USP19) (Fig. 3D). In addition, USP19 also enhanced abundances of intact 80-kDa V5-N298-GFP and its N-terminally-cleaved 55-kDa polypeptide, but this enhancement appeared to be also reduced by USP19 $19^{\triangle T M}$ to the control levels (Fig. 3E). These demonstrate that deubiquitination of Nrf1 (and/or N298) by USP19 occurs in close proximity to their co-tethered ER membranes. However, it is, to our surprise, that USP19 enhanced abundances of V5-N298 ${ }^{\Delta 2-36}$-GFP and its N-terminally-cleaved $55-k D a$ polypeptide, and such enhancement was also abolished by USP19 ${ }^{\triangle \mathrm{TM}}$. Collectively, these implicate that only membrane-tethered USP19 exerts its enzymatic activity to mediate deubiquitination of Nrf1, N298 or N298 ${ }^{\Delta 2-36}$ fusion proteins. Of note, a small fraction of N298 ${ }^{\Delta 2-36}$ fusion protein (lacking the N-terminal ER-anchored peptide of Nrf1) may still gain access to the membrane-tethered USP19, although it is largely located in the nucleus.
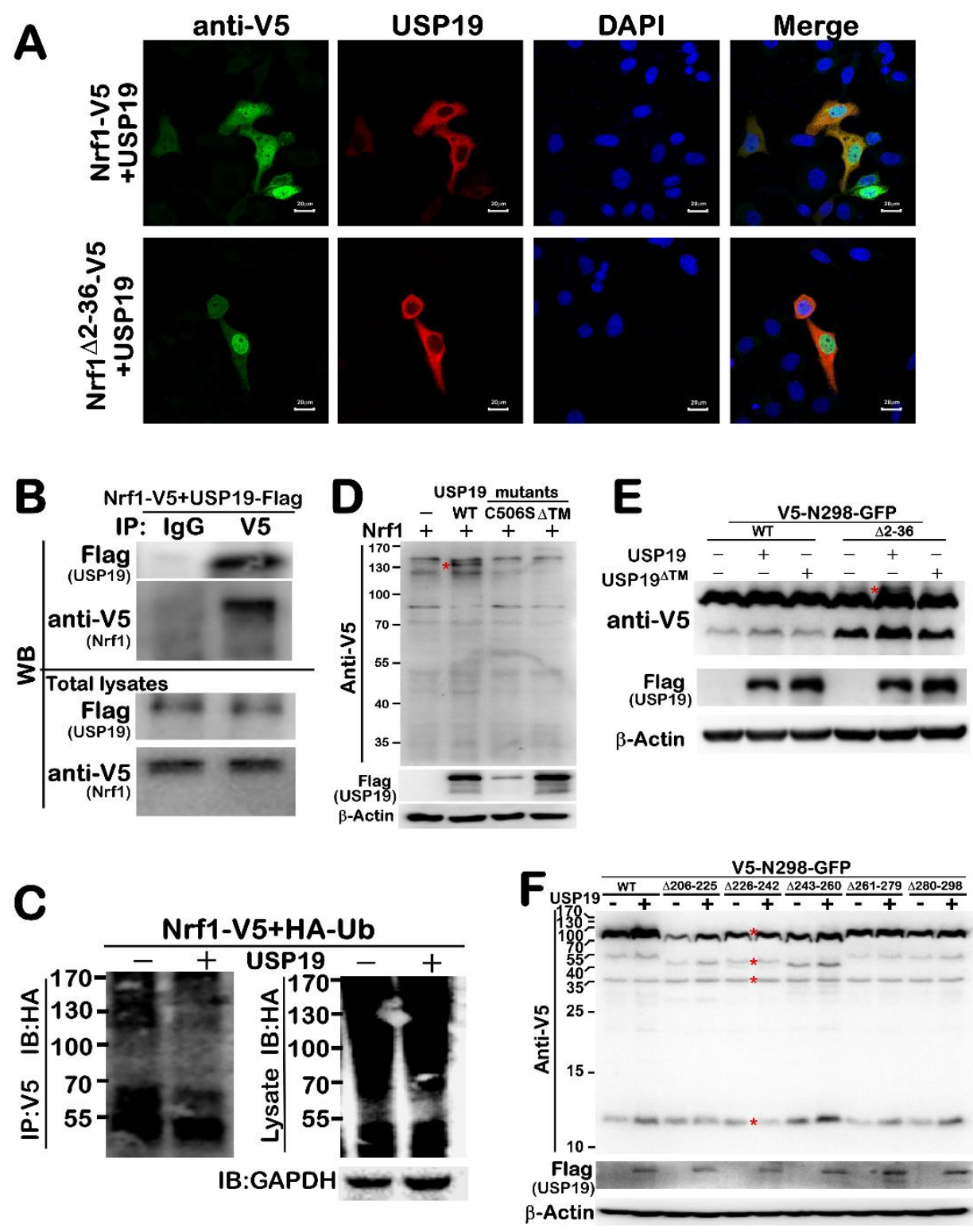
bioRxiv preprint doi: https://doi.org/10.1101/2020.10.05.326363; this version posted October 6, 2020. The copyright holder for this preprint (which was not certified by peer review) is the author/funder, who has granted bioRxiv a license to display the preprint in perpetuity. It is made available under aCC-BY-NC-ND 4.0 International license.

Figure 3. Deubiquitination of Nrf1 by its interactor USP19 in their co-location close to the ER.

(A) After immunocytochemical staining of COS-1 cells co-expressing USP19 plus Nrf1 or Nrf1 ${ }^{\Delta 2-36}$ with antibodies against USP19 or V5 tag. distinct fluorescence images were achieved and also merged (scale bar $=20 \mu \mathrm{m}$ ).

(B) Immunoprecipitates (IP) of COS-1 cells co-expressing USP19-flag and Nrf1-V5 with anti-IgG or anti-V5 antibodies were analyzed by immunoblotting with anti-Flag or anti-V5 antibodies, respectively (upper two panels). Besides, the inputs of whole-cell lysates were examined in parallel experiments (lower two panels).

(C) Another anti-V5 immunoprecipitates of COS-1 cells co-expressing both of Nrf1-V5 and HA-Ub, plus USP19 or not, were visualized by anti-HA immunoblotting (left panel). The whole-cell lysates were examined (right panels).

(D) Total lysates of COS-1 cells co-transfected with an expression construct for Nrf1 alone or plus USP19 (WT), its mutants USP19 ${ }^{\mathrm{C506S}}$ or USP19 ${ }^{\triangle \mathrm{TM} M}$ were determined by Western blotting with antibodies against USP19 or V5 tag.

(E) Two sandwiched chimeric proteins V5-N298-GFP and V5-N298 ${ }^{\Delta 2-36}$-GFP were allowed for co-expression with USP19, USP $19^{\triangle \mathrm{TM}}$ or neither, before immunoblotting with V5 or USP19 antibodies.

(F) Expression of V5-N298-GFP and its deletion mutants were also further evaluated by Western blotting with V5 or USP19 antibodies (also see Figure S3).

Distinct contributions of various N298 peptides to USP19-enhanced abundances of Nrf1 and its processing

To gain in-depth insight into distinct contributions of various N298 peptides to USP19-enhanced stability of Nrf1 and its proteolytic processing, we created a series of expression constructs by mutagenesis mapping of the N298 region within V5-N298-GFP (Figs. 3F and S3). The abundances of intact full-length V5-N298-GFP of 80-kDa and its three major N-terminal cleaved polypeptides of 55-kDa, 35-kDa and 12.5-kDa were strikingly enhanced by USP19 (Figs. 3F and S3). However, such USP19-triggered effects were abolished by either of the mutants V5-N298 ${ }^{\Delta 2-10}$-GFP (lacking aa 2-10 of Nrf1 facing the cytoplasmic side, Fig. S3A) and V5-N298 ${ }^{\Delta 226-242}$-GFP (lacking aa 226-242 of Nrf1 containing the ETGE motif, Fig. 3F), but almost unaffected by other mutants examined (Figs. 3F and S3). These imply that aa 2-10 and 226-242 within Nrf1 may serve as USP19-binding sites or its enzyme-targeting sites.

Apart from aa 2-10 and 226-242 of Nrf1, its mutants of other peptides within N298 remained to be subject to regulation by USP19 (Fig. 3F and S3), no matter whether they are selectively processed through topovectoriallyregulated juxtamembrane proteolysis (Xiang et al., 2018a). Of note, aa 11-22 of Nrf1 (i.e., the core h-region of NHB1 signal peptide that enables it to anchor within the ER membrane) were deleted to yield V5-N298 ${ }^{\Delta 11-22}$-GFP, such that its smaller N-terminally-cleaved polypeptides of between 35-kDa and 12.5-kDa, but not its longer 55-kDa polypeptide, appeared to be unaltered by USP19 (Fig. S3A). By contrast, the N-terminal 12.5-kDa polypeptide of Nrf1 was abolished by deletion of its NHB2-adjoining peptides (to yield other two mutants V5-N298 ${ }^{\Delta 81-106-G F P}$ and V5-N298 ${ }^{\Delta 107-124}$-GFP), whilst the abundances of their N-terminal 35-kDa polypeptides were unaffected by USP19 (Fig. S3B). In addition, deletion of the DIDLID/DLG-adjoining aa 125-170 and 171-186 yielded two unstable mutants of V5-N298 ${ }^{\Delta 81-106}$-GFP and V5-N298 ${ }^{\Delta 107-124}$-GFP, such that both fusion proteins were subjected to rapidly proteolytic processing to give rise to a major 70-kDa or 40-kDa polypeptides, respectively, along with continuously processed small polypeptides, but they were still enhanced by USP19 (Fig. S3B). Collectively, these results demonstrate that such discrete effects of USP19 on Nrf1 abundance and its N-terminal processing are modulated selectively through its membrane-topological mechanism, as described by (Xiang et al., 2018a; Zhang et al., 2015; Zhang et al., 2014).

Putative deubiquitination of Nrf1 by USP19 contributes to its stability and processing

Ubiquitination of Nrf1 was considered to occur prior to, and be essential for, its proteolytic processing to yield several cleaved polypeptides (Sha and Goldberg, 2014). But, our previous work showed that putative ubiquitination of Nrf1 (at Lys $5,6,70$ in its NTD and/or Lys ${ }^{169,199,205}$ in its AD1, Fig. 4A) is not a prerequisite for retro-translocation of this CNC-bZIP protein by $\mathrm{p97}$, before being subjected to its selective processing by cytosolic proteases (Xiang et al., 
2018a). This controversial issue has led us to test contribution of Nrf1 deubiquitination to its proteolytic processing. As anticipated, co-expression of both USP19 and Nrf1 enabled the latter CNC-bZIP protein to give rise to an obvious deubiquitinated isoform (resolved by $10 \%$ SDS-PAGE gels in pH8.3 Tris-Glycine running buffer, Figs. 1B \& 4B1). Such a similar USP19-deubiquitinating isoform of Nrf1 had also emerged directly from the mutant Nrf1 ${ }^{6 \times K / R}$ (in which all six potential ubiquitin-conjugated lysines were substituted by arginines), and its abundance were unaffected by this protease (Figs. 4B1, 4C1). However, the deubiquitinating isoform of $\mathrm{Nrf1}{ }^{6 \times K / R}$ was, to varying degrees, diminished or even abolished by recovery of the indicated lysines from this mutant (e.g., Nrf1 ${ }^{\mathrm{K} 70+5 \times K / R}$ made from the K70 recovery from Nrf1 ${ }^{6 \times K / R}$ ) (Figs. 4B1 and S4A). Furtherly, $\mathrm{Nrf1}^{\mathrm{K} 70 \mathrm{R}}$, but not Nrf1 ${ }^{\mathrm{K} 5 / 6 \mathrm{R}}, \mathrm{Nrf1}{ }^{\mathrm{K} 169 \mathrm{R}}, \mathrm{Nrf1}{ }^{\mathrm{K} 199 \mathrm{R}}$ or Nrf1 ${ }^{\mathrm{K} 205 \mathrm{R}}$, gave rise to a similar deubiquitinated isoform to that arising from Nrf1 ${ }^{6 \times K / R}$, but its abundance was substantially highlighted by USP19 (Figs. 4C1 and S4C). By contrast, putative USP19-deubiquitinating isoform of Nrf1 ${ }^{\mathrm{K} 5 / 6 \mathrm{R}}, \mathrm{Nrf1}{ }^{\mathrm{K} 169 \mathrm{R}}, \mathrm{Nrf1}{ }^{\mathrm{K} 199 \mathrm{R}}$ or $\mathrm{Nrf1}^{\mathrm{K} 205 \mathrm{R}}$ was presented only after co-expressing this protease. Collectively, these indicated that deubiquitination of Nrf1 by USP19 occurs at all six lysine residues, of which the K70 serves as a key site for this protease.

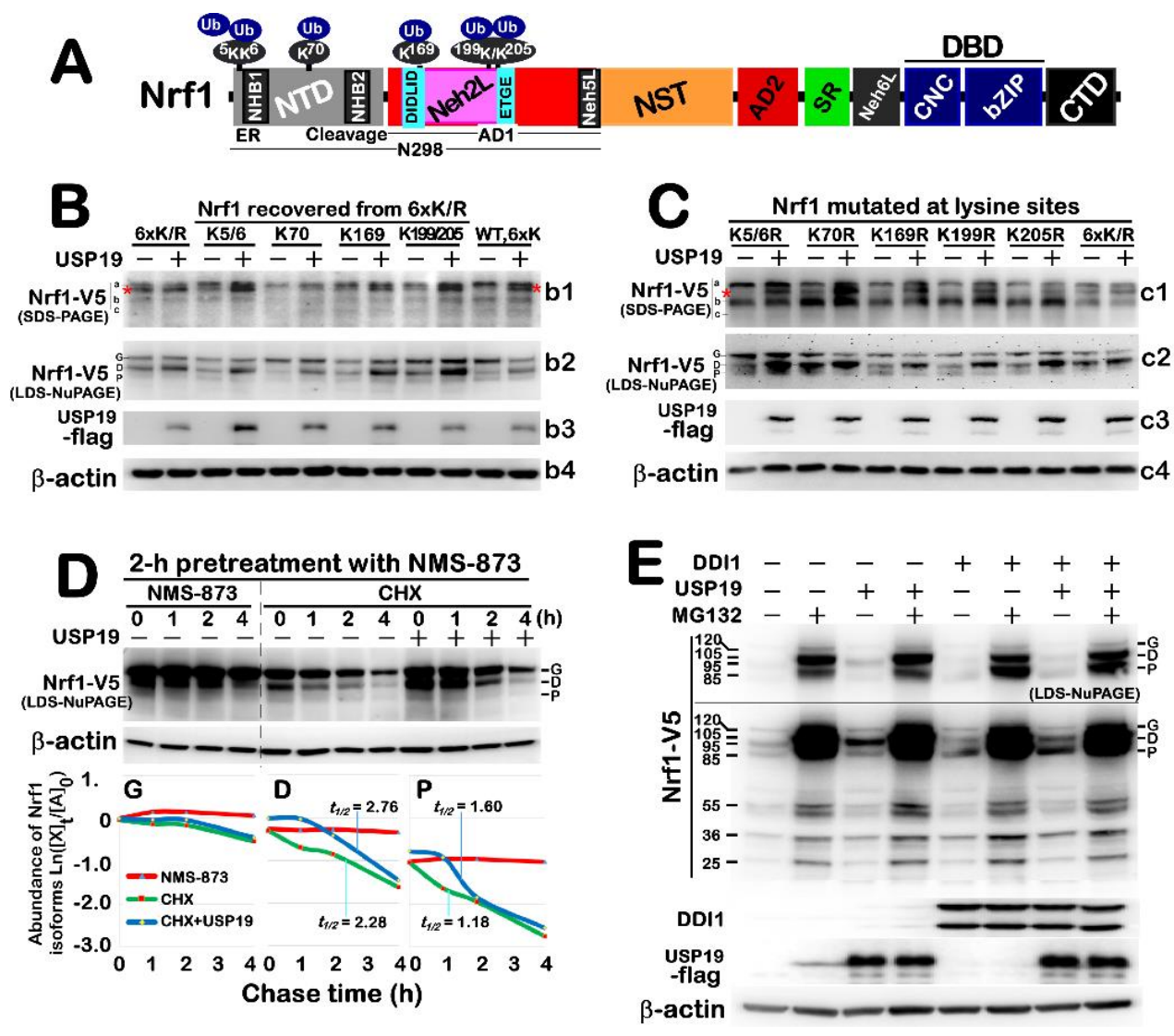

Figure 4. Putative deubiquitination of Nrf1 by USP19 contributes to its stability and processing.

(A) Schematic of distinct functional domains in Nrf1. Within its NTD, three putative ubiquitin-conjugated lysines are situated close to its NHB1-adjoining ER-targeting or NHB2-adjacent cleavage sequences. Another three lysines are located within AD1. The N298 portion of fusion proteins is composed of both NTD and AD1.

(B) Total lysates of COS-1 cells that had been transfected with expression constructs for wild-type Nrf1 (WT, 6×K), its mutant $\mathrm{Nrf1}^{6 \times K / R}$ (i.e., $6 \times \mathrm{K} / \mathrm{R}$ ) or one of those recovered variants (i.e., K5/6, K70, K169 and K199/K205) from the $\mathrm{Nrf1}^{6 \times K / R}$ mutant, plus USP19 or not, were separated by $8 \%$ SDS-PAGE gels or 4-12\% LDS-NuPAGE gels in distinct running buffers, and visualized by immunoblotting with antibodies against V5 tag or USP19, respectively.

(C) Western blotting of COS-1 cell lysates expressing one of distinct lysine-to-arginine mutants of Nrf1 (i.e. K5/6R, K70R, K169R, K199R, K205R and 6×K/R) alone or plus USP19 was carried out as described above. 
bioRxiv preprint doi: https://doi.org/10.1101/2020.10.05.326363; this version posted October 6, 2020. The copyright holder for this preprint (which was not certified by peer review) is the author/funder, who has granted bioRxiv a license to display the preprint in perpetuity. It is made available under aCC-BY-NC-ND 4.0 International license.

(D) COS-1 cells expressing Nrf1 alone or plus USP19 were pretreated for $2 \mathrm{~h}$ with NMS-873 (10 $\mu \mathrm{mol} / \mathrm{L})$ and then treated with $\mathrm{CHX}(50 \mu \mathrm{g} / \mathrm{ml})$ for indicated lengths of time, before being harvested. The cell lysates were resolved by $4-12 \%$ LDS-NUPAGE gels and visualized by Western blotting as described above. Subsequently, the intensity of three major immunoblots representing Nrf1 polypeptides was quantified, as shown graphically (in the bottom).

(E) Western blotting analysis of COS-1 cells that had been allowed for expressiong of Nrf1 alone or plus USP19 and/or DDI1, and then treated for $2 \mathrm{~h}$ with MG132 (+ $5 \mu \mathrm{mol} / \mathrm{L})$ or not (-) was carried out as described above.

Further examination of protein separation by 4-12\% LDS-NuPAGE in pH7.7 MOPS running buffer) revealed that USP19 enables wild-type Nrf1 glycoprotein to be reduced, instead of its deglycoprotein being enhanced by this protease (Figs. 4B2 and S4B). By contrast, the glycoprotein abundances of Nrf1 ${ }^{6 \times K / R}$ and other mutants appeared to be not or less affected by USP19, but their deglycoprotein abundances were increased to varying extents (Figs. 4B2, $4 C 2$, S4B and S4D). Of note, a relative stronger deglycoprotein of Nrf1 ${ }^{\mathrm{K} 70 \mathrm{R}}$ was generated in the absence of USP19, whilst a similar deglycoprotein of $\mathrm{Nrf1}^{\mathrm{K} 70+5 \times \mathrm{K} / \mathrm{R}}$ was almost prevented by the $\mathrm{K} 70$ recovery from Nrf1 ${ }^{6 \times K / R}$, but their abundances were still augmented by USP19 (Figs. 4B2 \& 4C2). Together, these suggest that the stability of Nrf1 and its processing (particularly at K70) may be altered by USP19-mediated deubiquitination.

To clarify a role of USP19 in p97-driven retrotranslocation of Nrf1 and its subsequent proteolytic processing by cytosolic proteases, we performed a pulse-chase experiment of COS-1 cells that had been allowed for expression of Nrf1 alone or plus USP19, and then pretreated with a p97-specific inhibitor NMS-873 for $2 \mathrm{~h}$, before addition of 50 $\mu \mathrm{g} / \mathrm{ml}$ of $\mathrm{CHX}$. As excepted, inhibition of p97-mediated repositioning of Nrf1 into extra-ER compartments led to an accumulation of this CNC-bZIP glycoprotein as accompanied by a gradual reduction of its deglycoprotein (Figs. 4D \& S4E, lanes 1-4). Subsequently, the recovery of p97 from its inhibition by NMS-873, at the same time when newlysynthesized proteins were blocked by $\mathrm{CHX}$, rendered the existing ER-located Nrf1 to be dynamically dislocated into the cytoplasmic side of membranes, whereupon it was subjected to its successive processing. The results revealed that time-lapse conversion of Nrf1 glycoprotein was unaffected by USP19 (Figs. 4D \& S4E, lanes 5-12) as showed graphically (Fig. 4D, lower left panel). By contrast, turnover of Nrf1 deglycoprotein and its processed isoform was prolonged by USP19, which was determined by obvious changes in both half-lives that were respectively extended from 2.28 to 2.76 and from 1.18 to $1.60 \mathrm{~h}$ after $\mathrm{CHX}$ treatment, when compared with those equivalents without USP19 (Fig. 4D, lower middle and right panels). This demonstrates that deubiquitination of Nrf1 by USP19 occurs after p97-driven dislocation of this CNC-bZIP protein to yield its deglycoprotein and rescues from being degraded by proteasomes and/or other cytoplasmic proteases (e.g. DDI1).

Co-transfection experiments of Nrf1 with USP19 or DDI1 alone or both together unraveled that this CNC-bZIP protein is subjected to the proteolytic processing by DDI1 (Fig. 4E). Notably, USP19 enhanced abundances of Nrf1 deglycoprotein and processed protein, but this processed protein was increased by DDI1, as accompanied with an instead decrease in deglycoprotein of Nrf1 (Fig. 4E).

\section{USP19 exerts a biological function required for Nrf1-governed proteostasis and tumorigenesis}

Since deubiquitination of Nrf1 by USP19 confers it to be rescued from the ubiquitin proteasomal degradation, this CNC-bZIP factor is accumulated and allowed for transcriptional regulation of proteasomal subunits by a similar way to the 'bounce-back' response to limited proteasomal inhibition(Steffen et al., 2010). Thus, to explore such a similar biological role of USP19 in regulating Nrf1, we here examined distinct transcriptional expression of its target

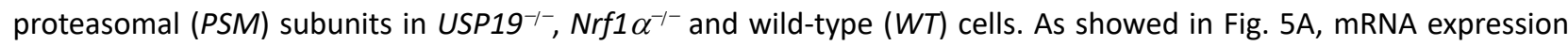
levels of six examined genes (PSMA1, PSMB5, PSMB6, PSMB7, PSMC6 and PSMD12) were significantly suppressed in $U S P 19^{-1-}$ cells, to varying extents that are roughly similar to those measured from $\mathrm{Nrf1} \alpha^{-/-}$cells. Besides, similar 
decreases in protein expression levels of three core enzymatic subunits (PSMB5, PSMB6, PSMB7) were determined in

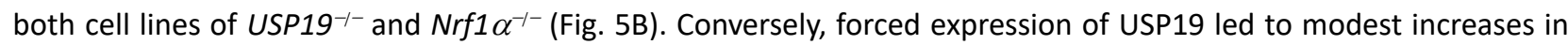
mRNA expression levels of some proteasomal subunits (Fig. S5A).
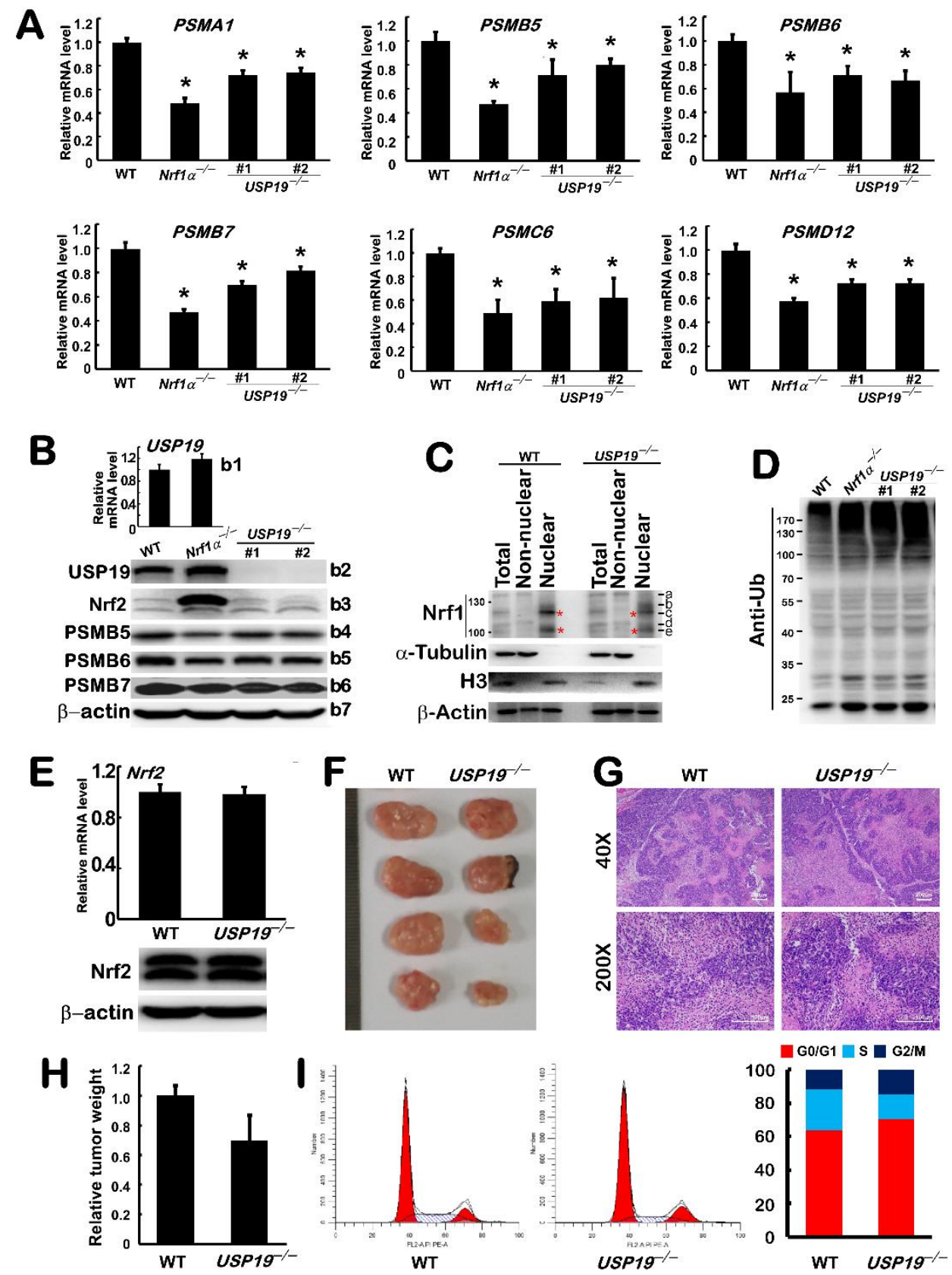

Figure 5. A biological function of USP19 is required for Nrf1-governed proteostasis and tumorigenesis

(A) Transcriptional expression of Nrf1-target proteasomal subunits PSMA1, PSMB5, PSMB6, PSMB7, PSMC6 and PSMD12 in

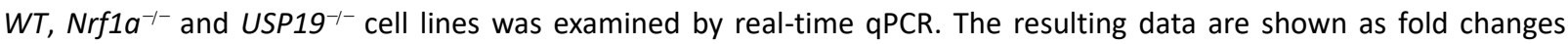
(mean $\pm \mathrm{SEM}, \mathrm{n}=3 \times 3$ ) with significant decreases $(* p<0.01)$ as compared to the controls.

(B) Top panel shows real-time qPCR analysis of mRNA expression levels of USP19 in WT and Nrf1 $a^{-1-}$ cells. The lower panels manifest Western blotting of $\mathrm{WT}, \mathrm{Nrfl}^{-a^{--}}$and $\mathrm{USP} 19^{-{ }^{-}}$cell lysates with distinct antibodies against USP19, Nrf2, PSMB5, PSMB6 or PSMB7, respectively.

(C) Subcellular fractions of WT and USP19-/- cells were determined by immunoblotting with the indicated antibodies.

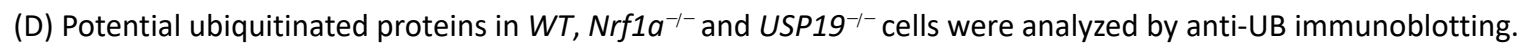


bioRxiv preprint doi: https://doi.org/10.1101/2020.10.05.326363; this version posted October 6, 2020. The copyright holder for this preprint (which was not certified by peer review) is the author/funder, who has granted bioRxiv a license to display the preprint in perpetuity. It is made available under aCC-BY-NC-ND 4.0 International license.

(E) Both mRNA and protein expression levels of Nrf2 in WT and USP19-1- cells were detected as described above.

(F) Two distinct phenotypes of xenograft tumors in nude mice were derived from subcutaneous inoculation $W T$ and USP19-/- hepatoma cell lines in nude mice.

(G) The pathohistological photographs of the above xenograft tumors were achieved after HE staining. (Scale bar $=200 \mu \mathrm{m}$ in $\times 40$ or $100 \mu \mathrm{m}$ in $\times 200$ ).

(H) Relative weights of xenograft tumors derived from WT and USP19-/- cells were calculated as shown graphically.

(I) Flow cytometry analysis of WT and USP19-1- cell cycles was illustrated (left panel). The data of three independent experiments $(n=3)$ are calculated and shown as distinct columns (right panel).

Subcellular fractionation revealed that, apart from the full-length glycoprotein-A of Nrf1 in the cytoplasmic non-nuclear fraction, all Nrf1-processed isoforms-B to -E was recovered predominantly in the nuclear fraction, but obviously reduced in USP19-/- cells, when compared with WT cells (Figs. 5C and S5B). Thereby, it is inferable that knockdown of USP19 causes a marked decrease in the processed active Nrf1 and results in reduced expression of proteasomal subunits. This is supported by further experiments, showing evident accumulation of ubiquitinated

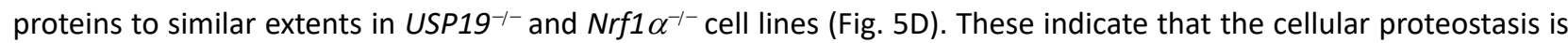
disrupted in USP19 ${ }^{--}$cells by dysfunction of proteasomal proteolytic degradation of ubiquitinated proteins. Such being the case, USP19-1- cells displayed a weaken resistance to cytotoxicity of the proteasomal inhibitor bortezomib (BTZ) , whilst $\mathrm{Nrf1} \alpha^{-1-}$ cells were embodied with more vulnerability to BTZ, when compared to WT cells (Fig S5C).

By contrast, mRNA and protein levels of Nrf2 were unaltered in USP19-1- cells (Fig. 5E). Further investigation of xenograft model mice showed that USP19-1--derived tumor growth was modestly reduced, when compared to WT control mice (Fig. 5F). The tumor pathohistochemistry revealed a lower tumorigenicity of USP19 ${ }^{-l_{-}}$cells than that of its parent WT HepG2 cells (Fig. 5G, 5H). Subsequently, flow cytometry unraveled that USP19-1- cell cycle seemed to be arrested at its prolonged G0/G1 phase, instead of its shorter S phase (Figs. 5I), but no changes in apoptosis of this cell lines were observed by comparison with WT cell controls (Fig. S6A). Taken altogether, these demonstrate that USP19 plays a biological role required for Nrf1-governed proteostasis and tumorigenesis.

Transcriptional expression of USP19 is mediated by Nrf2, as well by Nrf1, at distinct layers

Interestingly, we found a significant diminishment in mRNA and protein expression levels of USP19 in Nrf2 ${ }^{-1-}$ cells (Fig. 6A). In turn, over-expression of Nrf2 enabled USP19 expression to be significantly increased (Fig 6B). This implies the transcriptional regulation of USP19 by Nrf2. To address this, a USP19-Luc (i.e., its gene promoter-driven luciferase) reporter was created and subjected to co-transfection with an Nrf2 expression construct. As anticipated, the results revealed that transcriptional activity of USP19-Luc reporter was elevated by Nrf2 (Fig. 6C, upper panel). Notably, ectopic over-expression of Nrf1 also stimulated induction of USP19-Luc transcription (Fig. 6C, lower panel). However, loss of $N r f 1 \alpha$ also caused a modest increase in the expression of USP19 per se; this is thus deduced to be resulted from aberrant hyper-expression of $\mathrm{Nrf2}$ in $\mathrm{Nrf1} \alpha^{-1-}$ cells (Figs. 5B, S6B). Therefore, it is inferable that bona fide transcription of the USP19 gene regulated by Nrf2 is suppressed to a considerable lower extent in $N r f 1 \alpha^{-/-}$cells. Similar inhibitory results were also confirmed by transcriptomic analysis of $\mathrm{Nrf1} \alpha^{-1-}$ and $\mathrm{Nrf2} 2^{-1-}$ cell lines, showing almost no changes in the mRNA expression of USP19 in Nrf1 $\alpha^{-/-}$cells, but as accompanied by a significant decrease in the expression of USP19 in Nrf2 ${ }^{-1-}$ cells (Fig. S6C). In addition, $\mathrm{Nrf1} \alpha^{-/-}$-led proteasomal dysfunction also resulted in an apparent accumulation of both Nrf2 and USP19 (Figs. 5B and S6B). Collectively, these indicate that differential expression of USP19 is mediated by such two CNC-bZIP factors Nrf1 and Nrf2 at distinct layers. 

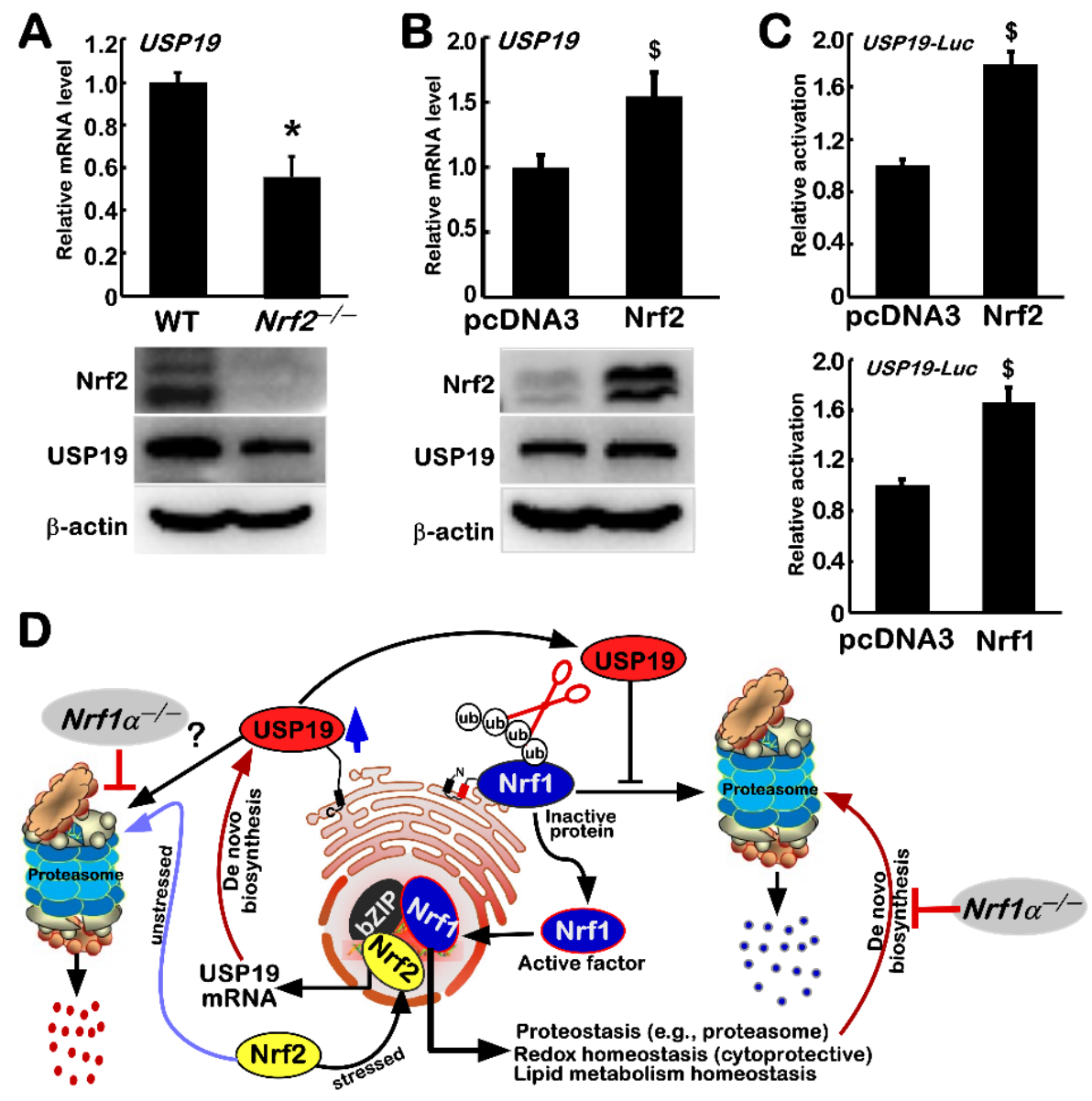

Figure 6. Transcriptional regulation of USP19 by Nrf2, as well by Nrf1

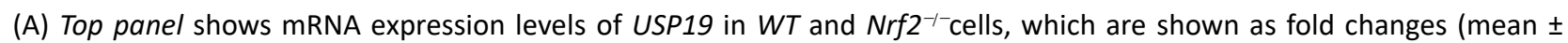
SEM, $n=3 \times 3$ ) with a significant decrease $(* p<0.01)$. Their protein expression levels were determined by Western blotting with antibodies against USP19 or Nrf2 (middle two panels).

(B) HepG2 cells expressing Nrf2 or not were subjected to examination of USP19 mRNA expression levels as shown as mean $\pm \operatorname{SEM}(n=3 \times 3)$ with a significant increase $(\$, p<0.01)$ (upper panel). Both protein abundances of USP19 and Nrf2 (middle two panels) were determined by Western blotting as described above.

(C) COS-1 cells, that had been co-transfected with both USP19-luc and $p R L-T K$ reporters, together with an expression construct for Nrf2 (upper panel), Nrf1 (lower panel) or an empty pcDNA3 vector, were allowed for 24-h recovery from transfection before the luciferase activity was measured. The resulting data are calculated as fold changes (mean \pm SEM, $n=3 \times 3)$ with significant increases $(\$, p<0.01)$ relative to the controls.

(D) A model is proposed to provide a better explanation of the Nrf2-USP19-Nrf1-Nrf2 axes along with their inter-regulatory feedback circuit, in order to maintain the robust steady-state of cell proteostasis, beyond redox homeostasis.

Scrutiny of our transcriptomic results revealed that most of five deubiquitinase families are regulated by Nrf1 and/or Nrf2 (Fig. S6C). It was found that COPS5, PRPF8, USP2, USP39, PSMD7 and PSMD14 were down-regulated, as accompanied by up-regulated OTUD1, USP4, USP20, USP21 and USP35 in Nrf1 $\alpha^{-/-}$cells, but they were unaltered in $\mathrm{Nrf2}^{-1-}$ cells, implying these are Nrf1-target genes. By contrast, additional 14 genes, including UCHL5, STAMBPL, USP1, USP7, USP11, USP12, USP16, USP18, USP24, USP28, USP32, USP33, USP34 and USP48, were up-regulated in $\mathrm{Nrf2} 2^{--}$cells, but unaffected in $\mathrm{Nrf1} \alpha^{-1-}$ cells, indicating they are Nrf2-specific target genes. Furtherly, comparison of 
bioRxiv preprint doi: https://doi.org/10.1101/2020.10.05.326363; this version posted October 6, 2020. The copyright holder for this preprint (which was not certified by peer review) is the author/funder, who has granted bioRxiv a license to display the preprint in perpetuity. It is made available under aCC-BY-NC-ND 4.0 International license.

USP14, USP15 and UPS25 that were up-expressed in Nrf2 $2^{--}$cells, but down-expressed in $\mathrm{Nrf1}^{-\alpha^{--}}$cells retaining hyper-active Nrf2, indicated that they are predominantly negatively regulated by Nrf2, rather than Nrf1. Another comparison of up-expression of COPS6 in Nrf2-elevated $\mathrm{Nrf1} \alpha^{-/-}$cells, but with its down-expression in $\mathrm{Nrf2} 2^{-/-}$cells uncovered positive regulation of this gene by Nrf2, but not Nrf1. Rather, down-regulation of USP5 and USP36 in $\mathrm{Nrf1} \alpha^{-1-}$ and $\mathrm{Nrf2}^{-1-}$ cell lines suggested that both are transcriptionally mediated by Nrf1 and Nrf2. Overall, these demonstrate there exists a complex hierarchical network, which is composed of Nrf1 and Nrf2, plus USP19 and other deubiquitinases, together with the ubiquitin-mediated proteasome system, all of which are major key nodes interlaced in dual positive and negative feedback circuits, in order to maintain normal robust proteostasis.

\section{Discussion}

After the NHB1 signal peptide of Nrf1 enables it to be anchored by a proper topology within the ER membranes, some portions of its other functional domains are translocated into the lumen, in which it is allowed for $\mathrm{N}$-linked glycosylation by the luminal-resident active center of hetero-oligometic oligosaccharyltransferase (OST) (Xiang et al., 2018b; Zhang et al., 2007) . During retrotranslocation of Nrf1 glycoprotein into the cytoplasmic side of membranes, its subsequent deglycoprotein is further modified by ubiquitination and ensuing deubiquitination, and all of these modified proteins are also subjected to the selective topovectorial regulation in their juxtamembrane proteolytic processing to yield multiple distinct isoforms (Xiang et al., 2018a; Zhang et al., 2015; Zhang et al., 2014). Once deglycoprotein of Nrf1 by peptide:N-Glycanases (PNGase) is dislocated from the ER and transferred into the nucleus, it can function as a bona fide active form to regulate transcriptional expression of its cognate target genes (Zhang et al., 2009; Zhang et al., 2014). Similar results have also been obtained from the Caenorhabditis elegans homologue Skn-1 (Lehrbach et al., 2019).

Nrf1 is regulated by USP19 through its deubiquitination in close proximity to the ER.

In close vicinity to the ER, Nrf1 is also subjected to ubiquitination by Hrd1 and other ubiquitin E3 ligases, before being targeted for the ubiquitin-mediated proteasomal degradation, in particular normal conditions(Steffen et al., 2010; Tsuchiya et al., 2011; Xiang et al., 2018b). Of note, dislocation of Nrf1 from the ER membranes is largely unaffected by its ubiquitination (Xiang et al., 2018a) and ensuing deubiquitination (in this study). Rather, it should be noted that the selective proteolytic processing of Nrf1 appears to be regulated by its putative deubiquitination of Nrf1 by USP19, but not by USP14 or USP15, because our evidence has uncovered that this deubiquitinase has an ability to augment the stability of its deglycoprotein and other processed proteins (Fig. 6D).

Herein, we found that two membrane-tethered proteins USP19 and Nrf1 are co-localized around and within the ER, such that this deubiquitinase can directly interact with the CNC-bZIP protein in close proximity to membranes. The interaction of both proteins appears to depend on two regions of Nrf1 between amino acids 2-10 and/or 226-242. The former residues 2-10 of Nrf1 comprise the N-region of its ER-anchored NHB1 peptide, which faces the cytoplasm, where it is allowed for potential interaction with the ubiquitin-specific protease USP19. By contrast, the residues 226-242 of Nrf1 are generally buried in the ER lumen and thus segregated from cytosolic USP19 (Zhang et al., 2015; Zhang et al., 2014). Thereby, only after this ETGE-containing region within Nrf1 will be dynamically moved out of membranes to enter the cytoplasm, it can enable the CNC-bZIP protein to interact with USP19.

Further evidence has been provided, showing that deubiquitination of the ER-resident Nrf1 by USP19 confers it to be emerging as an deubiquitinated isoform. The deubiquitinating effect of USP19 on Nrf1 (or its N298 region) is completely abolished by either USP19 ${ }^{\mathrm{C506S}}$ (a mutant of its enzymatic active center) or USP19 ${ }^{\Delta \mathrm{TM}}$ (a deletion mutant of its C-terminal transmembrane domain). This indicates that only the membrane-anchored USP19 gains access to the ER-resident Nrf1, before its deubiquitinating reaction with this protease. Moreover, another lines of supportive evidence have been obtained from our experiments, unraveling that deubiquitination of Nrf1 by USP19 occurs only 
bioRxiv preprint doi: https://doi.org/10.1101/2020.10.05.326363; this version posted October 6, 2020. The copyright holder for this preprint (which was not certified by peer review) is the author/funder, who has granted bioRxiv a license to display the preprint in perpetuity. It is made available under aCC-BY-NC-ND 4.0 International license.

after p97-driven retrotranslocation of the luminal CNC-bZIP glycoprotein into extra-ER compartments, in which it is subjected to deglycosylation, deubiquitination and/or other proteolytic processing by distinct cytosolic enzymes.

A similar USP19-deubiquitinating isoform of Nrf1 seems to arise from expression of Nrf1 ${ }^{6 \times K / R}$ alone, and this mutant deubiquitinating isoform is unaffected by this protease. This demonstrates that deubiquitination of Nrf1 by USP19 occurs at all six lysines examined (Fig. 4A). Further examinations of distinct mutants and relevant recovery from Nrf1 ${ }^{6 \times K / R}$ revealed that the Lys ${ }^{70}$ is a key site for ubiquitination of Nrf1 and ensuing deubiquitination by USP19, whilst other three sites at Lys ${ }^{169}$, Lys ${ }^{199}$ and Lys ${ }^{205}$ within the CNC-bZIP protein are required for its ubiquitination and deubiquitination. However, such a couple of reversible modifications depends on the topological locations of all the four lysine sites $\left(\right.$ Lys $^{70}$, Lys ${ }^{169}$, Lys ${ }^{199}$ and Lys ${ }^{205}$ ) within distinct contexts of Nrf1 or its mutants. This is due to the fact that Nrf1 ubiquitination and its deubiquitination by USP19 occur only after dynamic repositioning of these lysines from ER luminal side of membranes into the cytoplasmic side.

Notably, Nrf1 ${ }^{\Delta 2-10}$ had been shown to give rise to a major strong glycoprotein, with little or none in its processed proteins (Zhang et al., 2015; Zhang et al., 2007). Similar results were also obtained from its N298 ${ }^{\Delta 2-10}$ fusion protein (Xiang et al., 2018a), which is endowed as a major full-length protein of $80-k D a$, but its proteolytic processing was substantially diminished by lacking the n-region of its NHB1 signal. The remnant N-terminally- processed proteins of between $55-\mathrm{kDa}$ and $12.5-\mathrm{kDa}$ from N298 ${ }^{\Delta 2-10}$ fusion mutant were, herein, found to be unaffected by USP19. These demonstrate that the Lys ${ }^{5 / 6}$-containing $n$-region of NHB1 signal is required for a proper membrane-topology of this CNC-bZIP protein folding within and around the ER and its subsequent repositioning into the cytoplasmic side of membranes. Hence, it is inferable that putative Lys ${ }^{5 / 6}$ ubiquitination of Nrf1 by Hrd1 and ensuing deubiquitination by USP19 are much likely to promote dynamic repartitioning of this CNC-bZIP protein into extra-ER compartments and subsequent proteolytic processing by cytosolic proteasome, DDI1/2 and other proteases.

The effect of USP19 on Nrf1 to erase its conjugated ubiquitins renders this CNC-bZIP protein to circumvent the ubiquitin-led proteasomal degradation, such that abundances of its deglycoprotein and derivative proteins (arising from the selective proteolytic processing by DDI1/2 and/or other proteases) are enhanced by this deubiquitinatase. However, this also manifests that the deubiquitinating effect of USP19 on Nrf1 processing is limited. As a matter of fact, the selective proteolytic processing of Nrf1 by cytosolic proteases (e.g., DDI1/2 (Koizumi et al., 2016; Lehrbach and Ruvkun, 2016; Xiang et al., 2018a)) is dictated by its intrinsic topologically-regulatory mechanism. This notion is established on the fact that distinct deletions of NHB1- and NHB2-adjoining peptides from Nrf1 or its N298 region result in a complete abolishment or significant diminishment of its $\mathrm{N}$-terminal putative DDI1/2-cleaved polypeptide with a variation in about 12.5-kDa molecular weight (Fig. S3). Such objective fact demonstrates that no DDI-specific cleavage sites exist within Nrf1, and even if doing so, this is determined by topovectorial processes of this CNC-bZIP protein to be repositioned into extra-ER side of membranes. This is further supported by another variation in the yield of other two N-terminally-cleaved polypeptides between 35-kDa and 55-kDa from the N298 fusion protein, in which their putative cleavage sites are deduced to be embodied within discrete peptide bonds of its AD1 domain. Within the N-terminal one-third of AD1, two DIDLID/DLG-adjoining elements (aa 125-170 \& 171-186) are required for the stability of Nrf1 or its N298 fusion proteins. The relevant mechanisms had been elucidated by (Widenmaier et al., 2017; Xiang et al., 2018a; Zhang et al., 2014).

\section{Nrf1 and Nrf2 have bidirectional inter-regulatory roles in co-targeting USP19 expression.}

Since USP19 enhances stability of deglycoprotein of Nrf1 and its processed proteins by escaping from ubiquintin proteasomal degradation, this is accompanied by an evident increase in the transcriptional activity of this CNC-bZIP factor to mediate expression of its target genes encoding proteasomal subunits. In turn, knockout of USP19 leads to an obvious decrease in the proteasomal expression, so that the resulting ubiquitinated proteins are accumulated in USP $19^{-/-}$cells. As such, USP19-/- cells still possess a modest resistance to cytotoxicity of the proteasomal inhibitor 
bioRxiv preprint doi: https://doi.org/10.1101/2020.10.05.326363; this version posted October 6, 2020. The copyright holder for this preprint (which was not certified by peer review) is the author/funder, who has granted bioRxiv a license to display the preprint in perpetuity. It is made available under aCC-BY-NC-ND 4.0 International license.

bortezomib, when compared with the equivalent of $\mathrm{Nrf1} \alpha^{-1-}$ cells (Fig. S5C). This implicates only partial inactivation

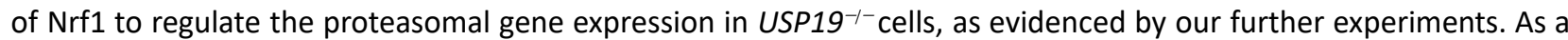
consequence, USP19-1--derived tumor growth in xenograft model mice was modestly retarded, when compared with wild-type controls. This is attributable to the arrest of USP19-/- cell cycle at its G0/G1 phase with a shortened S phase (in this study), whereas $\mathrm{Nrf1} \alpha^{-/-}$cell cycle is also arrested at its G2/M phase(Qiu et al., 2018; Ren et al., 2016). However, as $\mathrm{Nrf1} \alpha^{-/-}$-derived tumor growth is significantly incremented, it is further deteriorated and metastasized to the liver and lung in xenograft mice (Chen et al., 2020; Qiu et al., 2018; Ren et al., 2016). Such complete loss of Nrf1 $\alpha$-derived proteins results in an aberrant accumulation of Nrf2, as accompanied by inactivation of the tumor-repressor PTEN. By contrast, expression of Nrf2 at mRNA and protein levels is unaffected by partial inactivation of Nrf1 in USP19-/- cells. Taken altogether, these demonstrates that the remaining Nrf1's function can still be exerted as a dominant tumor-repressor, under such Nrf2-unaffected conditions in USP19 ${ }^{-1-}$ cells. Contrarily, hyper-active Nrf2 acts as a predominant functional rebel to become a cancer promoter, but it is successfully confined by the remnant Nrf1 in USP19-/- cells.

Within the regulatory feedback circuit, USP19 is positively regulated by Nrf2, but its transcriptional expression is only less or not promoted by accumulated Nrf2 in Nrf1 $\alpha^{-1-}$ cells. This implies that positive regulation of USP19 by Nrf2 appears to be suppressed by an not-yet-identified mechanism in Nrf1 $\alpha^{-1-}$ cells. In other words, Nrf1 $\alpha$-derived factors also indirectly contribute to positive regulation of USP19, albeit this protease is also subjected to a negative feedback loop governed by Nrf1-target proteasomal genes (Fig. 6D). As such, ectopic over-expression of Nrf1, like $\mathrm{Nrf2}$, leads to an increase in transcription activity of USP19-driven reporter. Such striking disparity in between effects of Nrf1 (and Nrf2) on endogenous USP19 and its promoter-driven reporter (i.e., USP19-LuC) genes indicates that their differential transcriptional expression could also depend on their genomic contexts of distinct topological settings.

In summary, this study demonstrates that USP19 serves as a novel mechanistic modulator of Nrf1, but not Nrf2. Differential transcriptional expression of this protease is regulated by Nrf2, as well by Nrf1, at distinct layers within bidirectional regulatory feedback circuits. In addition to USP19, most of distinct deubiquitinase family members are also, to greater or less degrees, regulated by Nrf1 or Nrf2 alone or both factors. Thereby, it is inferable that these key nodes are topologically organized together within a complex hierarchical regulatory network, in order to maintain and perpetuate the robust steady-state of distinct cellular homoestasis and subcellular organelle integrity.

\section{Materials and Methods}

Cell lines, Culture and Transfection

Knockout cell lines of USP19-/- were here created by CRISPR/Cas9-mediated gene manipulation on the basis of HepG2. Briefly, single guide RNAs targeting exons 3 of the USP19 gene (\#1, 5'-AGGAAGCCCGAACCAGAAGCGG-3'; \#2, 5'-ATGCATCAAACCGTGAGCAGCGG-3') were cloned into a pCAG-T7-cas9+gRNA-pgk-Puro-T2A-GFP vector (Viewsolid Biotech, Beijing, China). The plasmid was then transfected into HepG2 cells with Lipofectamine 3000 (Invitrogen, Carlsbad, CA), followed by selecting positive cells with puromycin $(2.5 \mu \mathrm{g} / \mathrm{ml})$. The anti-drug monoclonal cells (all of which had been originated from a single cell) were identified by sequencing the specific genomic DNA locus. The

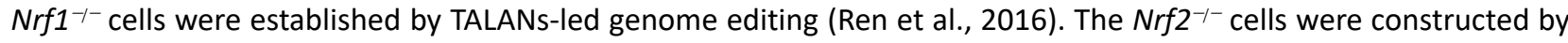
CRISPR/Cas9-editing system(Qiu et al., 2018). These cell lines were maintained in DMEM supplemented with $5 \mathrm{mM}$ glutamine, $10 \%(\mathrm{v} / \mathrm{v})$ foetal bovine serum (FBS), 100 units $/ \mathrm{mL}$ of either of penicillin and streptomycin, in the $37^{\circ} \mathrm{C}$ incubator with $5 \% \mathrm{CO}_{2}$. In addition, some of these cell lines were transfected with the indicated constructs for $8 \mathrm{~h}$ by using the Lipofectamine ${ }^{\circledR 3} 3000$ agent mixed in the Opti-MEM (gibca, Waltham, MA, USA). The cells were then allowed for recovery from transfection in a fresh complete medium for $24 \mathrm{~h}$, before the other experiments were conducted as indicated. 
bioRxiv preprint doi: https://doi.org/10.1101/2020.10.05.326363; this version posted October 6, 2020. The copyright holder for this preprint (which was not certified by peer review) is the author/funder, who has granted bioRxiv a license to display the preprint in perpetuity. It is made available under aCC-BY-NC-ND 4.0 International license.

\section{Expression constructs}

Five expression constructs for human USP14, USP15, USP19, USP30 and USP48 were here created by inserting their full-length cDNA sequences into the p3xFlag-CMV-14 vector. Additional two mutants USP19 ${ }^{\triangle T M}$ and USP19C506s were also made. The former USP19 ${ }^{\triangle \mathrm{TM}}$ was constructed deleting a cDNA fragment encoding the transmembranerelevant residues 50-70 of USP19, while the latter USP19 ${ }^{\mathrm{C5065}}$ mutant was yielded by replacing its original cysteine at position 506 with a serine residue. Notably, 4202-bp of the USP19 promoter was cloned into the Kpnl/HindIII site of the PGL3-Basic vector to yield a USP19-Luc reporter. In addition, two expression constructs for human Nrf1 and Nrf2 were made by inserting the full-length cDNA sequences into the Kpnl/Xbal site of pcDNA3.1/V5His B, as described previously by (Zhang et al., 2006; Zhang et al., 2007). A set of mutants of those putative ubiquitinated lysine sites and relevant recovery plasmids from its mutation was created on the base of a Nrf1 expression construct. Another set of $\mathrm{N} 298$ fusion protein expression construct and its deletion mutants was also constructed by inserting the $\mathrm{N}$-terminal first 298-aa of Nrf1 into peGFP-N2 vector. Of note, the N-terminus of N298 was also tagged by attaching the V5 epitope, as described by(Xiang et al., 2018a).

\section{Real-time qPCR analysis of mRNA expression}

Approximately $2.5 \mu \mathrm{g}$ of total RNAs (obtained by using an RNeasy mini-RNAsimple Kit, Tiangen Biotech, Beijing, China) were added in a reverse-transcriptase reaction to generate the first strand of cDNA (by using the Revert Aid First Strand Synthesis Kit, Thermo, Waltham, MA, USA). The synthesized cDNA was served as the template for qPCR, in the GoTaq ${ }^{\circledR} \mathrm{qPCR}$ Master Mix (Promega, Madison, WI, USA), before being deactivated at $95^{\circ} \mathrm{C}$ for $10 \mathrm{~min}$, and then amplified by 40 reaction cycles of being annealed at $95^{\circ} \mathrm{C}$ for $15 \mathrm{~s}$ and then extended at $60^{\circ} \mathrm{C}$ for $30 \mathrm{~s}$. The final melting curve was validated to examine the amplification quality. Of note, the mRNA expression level of $\beta$-actin served as an optimal internal standard control, relative to other mRNA expression levels presented as fold-changes. All the forward and reverse primers of those indicated genes were shown in Table S1.

\section{Western blotting and pulse-chase experiments}

Experimental cells were harvested in a denatured lysis buffer $(0.5 \% \mathrm{SDS}, 0.04 \mathrm{~mol} / \mathrm{L} \mathrm{DTT}$, pH 7.5, containing 1 tablet of cOmplete protease inhibitor EASYpacks in $10 \mathrm{ml}$ of this buffer). The total lysates were further denatured by boiling at $100^{\circ} \mathrm{C}$ for $10 \mathrm{~min}$, sonicated sufficiently, and diluted with $3 \times$ loading buffer $(187.5 \mathrm{mmol} / \mathrm{L} \mathrm{Tris-} \mathrm{HCl}, \mathrm{pH} 6.8$, $6 \%$ SDS, 30\% Glycerol, $150 \mathrm{mmol} / \mathrm{L}$ DTT, 0.3\% Bromphenol Blue), before being re-boiled at $100^{\circ} \mathrm{C}$ for $5 \mathrm{~min}$. Thereafter, equal amounts of protein extracts were subjected to separation by either SDS-PAGE containing $8 \%$ or $10 \%$ polyacrylamide or LDS-NUPAGE containing 4-12\% polyacrylamide, in distinct pH running buffers, before being transferred to polyvinylidene fluoride (PVDF) membranes (Millipore, Billerica, MA, USA). The protein-transferred membranes were blocked for $1 \mathrm{~h}$ by $5 \%$ fat-free milk $(\mathrm{w} / \mathrm{v})$ resolved in PBS containing $0.1 \%$ Tween $20(\mathrm{v} / \mathrm{v})$. and then incubated with one of indicated primary antibodies for $2 \mathrm{~h}$ at room temperature or overnight at $4^{\circ} \mathrm{C}$, followed by washing steps. The antibody-incubated membranes were visualized by $2 \mathrm{~h}$ of re-incubation with an appropriate horseradish peroxidase-conjugated secondary antibodies, followed by enhanced chemiluminescence. To estimate the protein stability, its half-life was calculated by cycloheximide (CHX)-pulse chase experiments of distinct cell lines that had been transfected or not transfected with the indicated expression constructs, before being incubated with $50 \mu \mathrm{g} / \mathrm{ml} \mathrm{CHX}$ for an indicated time, followed by immunoblotting of these cell lysates.

\section{Luciferase Reporter Assay}

Equal numbers $\left(1.0 \times 10^{5}\right)$ of COS1 cells were allowed for growth in each well of 12 -well plates. After reaching $80 \%$ confluence, the cells were co-transfected for $8 \mathrm{~h}$ with an indicated luciferase plasmid alone or together with one of expression constructs in the Lipofectamine 3000 mixture, in which the Renilla-expressing pRL-TK plasmid served as an internal control for transfection efficiency. The cells were allowed for a recovery from transfection to be cultured 
in a fresh complete medium for $24 \mathrm{~h}$, before the luciferase activity was measured by the dual-reporter assay (Promega, Madison, WI, USA).

\section{Subcellular fractionation}

Equal numbers $\left(1 \times 10^{6}\right)$ of disitinct cell lines were allowed for growth in each of 6 -cm dishes for $24 \mathrm{~h}$ before being harvested by incubation with $1 \mathrm{~mL}$ of an ice-cold nuclei EZ lysis buffer (sigma, San Francisco, CA, USA). The total lysates were subjected to subcellular fractionation by centrifuging at $500 \times g$ for 5 min at $4^{\circ} \mathrm{C}$. The supernatants were collected and used as a non-nuclear cytoplasmic fraction, whilst the sediment fraction were further washed with the above lysis buffer for two times, each of which $0.5 \mathrm{~mL}$ of the nuclei EZ lysis buffer was added into the sediment fraction. Lastly, the nuclear pellets were obtained by centrifuging at $500 \times g$ for $5 \min$ at $4^{\circ} \mathrm{C}$. Subsequently, these fractions were further evaluated by Western blotting.

\section{Coimmunoprecipitation with ubiquitination analysis}

Experimental cells were lysed in $500 \mu \mathrm{L}$ of the NP-40 lysis buffer $(20 \mathrm{mM}$ Tris- $\mathrm{HCl}, \mathrm{pH} 7.4 \sim 7.5,150 \mathrm{mM} \mathrm{NaCl}, 1$ mM EDTA, 1\% NP-40, and 1 tablet of cOmplete protease inhibitor EASYpacks resolved in $10 \mathrm{ml}$ of this buffer). The lysates were centrifuged at $12,000 \mathrm{rpm}(=12396 \times \mathrm{g})$ for $10 \mathrm{~min}$ at $4^{\circ} \mathrm{C}$ to obtain the clear supernatants. Subsequently, the supernatants was subjected to immunoprecipitation by being incubated with $1.2 \mu \mathrm{g}$ of specific antibody overnight, and re-incubated with $20 \mu \mathrm{L}$ of Protein A/G PLUS-Agarose (santa cruz, Santa Cruz, CA, USA) at $4^{\circ} \mathrm{C}$ for $2 \mathrm{~h}$. After the beads were washed for three times, each with $1 \mathrm{ml}$ of the above lysis buffer containing a $500 \mathrm{mM}$ concentration of $\mathrm{NaCl}$, the resulting immunoprecipitates were resolved by SDS-PAGE gels and visualized by immunoblotting with indicated antibodies. For analysis of ubiquitination and deubiquitination status, the ubiquitin-expressing cell lysates were analyzed by Western blotting with its specific antibody or by immunoprecipitation with its HA-tag antibody.

\section{Immunocytochemistry with confocal microscopy}

Experimental cells $\left(3 \times 10^{5}\right)$, that had been allowed for overnight growth on a cover glass being placed in each well of 6-well plates, were co-transfected for $8 \mathrm{~h}$ with two distinct expression constructs for USP19 plus Nrf1 or its mutant and then allowed for $24 \mathrm{~h}$ recovery from transfection in the fresh complete medium. Thereafter, these cells were fixed for 15 min with 4\% paraformaldehyde in PBS buffer and then permeabilized for additional 10 min with $0.1 \%$ Triton X-100 in PBS. Subsequently, the fixed cells were subjected to immunocytochemistry by incubation with the primary antibodies against the V5-epitoped tag or USP19 (each with a dilution of 1:200) for $2 \mathrm{~h}$ and additional incubation with either Alexa Fluor 488-conjugeted Goat Anti-Mouse IgG $(\mathrm{H}+\mathrm{L})$ or TRITC-conjugeted Goat anti-Rabbit IgG $(\mathrm{H}+\mathrm{L})$ (both obtained from ZSGB-BIO, Beijing, China) for $2 \mathrm{~h}$ at room temperature in the dark, followed by an additional 5-min staining of DNA with DAPI (Beyotime, Beijing, China). The results of distinct fluorescence images were achieved by confocal microscopy.

\section{Subcutaneous tumor xenografts in nude mice with pathohistological analysis}

Mouse xenograft models were made by subcutaneous heterotransplantation of wild-type human hepatoma cells or its derived USP19-/- cells in nude mice. Briefly, equal amounts $\left(1 \times 10^{7}\right)$ of experimental cells growing in the exponential phase were suspended in $0.1 \mathrm{ml}$ of serum-free DMEM and then inoculated subcutaneously at a single site in the right upper back region of male nude mice (BALB/C nu/nu, 4-6 weeks, $18 \mathrm{~g}$ ). The procedure of injection into all the mice was completed within $30 \mathrm{~min}$. Subsequently, the formation of these murine subcutaneous tumor xenografts was observed successively until all the mice were sacrificed. The transplanted tumors were excised immediately after they were executed. The tumor sizes were also calculated by a standard formulate (i.e. $\left.V=a b^{2} / 2\right)$, as shown graphically ( $n=5$ per group). Furthermore, the tumor tissues were immersed in $4 \%$ paraformaldehyde 
overnight and then transferred to 70\% ethanol. The fixed tumor tissues were dehydrated by a serial gradient of, alcohol, embedded in paraffin wax blocks, and sectioned into a series of 5- $\mu \mathrm{m}$-thick slides. Before pathohistological staining, the sections were de-waxed in xylene and rehydrated by progressively-decreased concentrations of ethanol. Subsequently, the tissue sections were stained routinely by hematoxylin and eosin (H\&E) and visualized by microscopy. In addition, it should also be noted that these nude mice were purchased from the Laboratory Animal Center of Chongqing Medical University (with a certificate SCXK (YU) 2007-0001). They were maintained under the optimal conditions for hygiene, temperature and photoperiods (12L:12D), and allowed ad libitum to food and water, according to the institutional guidelines for the care and use of laboratory animals. All the experimental procedures were approved by the Ethics Committee of Chongqing medical University.

\section{Flow cytometry analysis of cell cycle and apoptosis}

Experimental cells $\left(6 \times 10^{5}\right)$ were allowed for growth in $60-\mathrm{mm}$ cell culture plate for $48 \mathrm{~h}$ and synchronization by 12-h starvation in a serum-free medium, before being treated with $10 \mu \mathrm{mol} / \mathrm{L} \mathrm{BrdU}$ for $12 \mathrm{~h}$. The cells were fixed for 15 min with $100 \mu \mathrm{L}$ of BD Cytofix/Cytoperm buffer (with a mixture of both the fixative paraformaldehyde and the detergent saponin) at room temperature and then permeabilized for 10 min within $100 \mu \mathrm{L}$ of BD Cytoperm permeabilization buffer plus (with fetal bovine serum served as a staining enhancer) on ice. Thereafter, the cells were treated for $1 \mathrm{~h}$ at $37^{\circ} \mathrm{C}$ with $100 \mu \mathrm{L}$ of DNase (at a concentration of $300 \mu \mathrm{g} / \mathrm{mL}$ in PBS) to allow for exposure to BrdU incorporation and then stained with FITC (fluorescein isothiocyanate) conjugated anti-BrdU antibody for 60 min at room temperature. Subsequently, the cells were suspended in $20 \mu \mathrm{L}$ of a 7-amino-actinomycin D solution for 20 -min DNA staining and re-suspended in $0.5 \mathrm{~mL}$ of another staining buffer (i.e., $1 \times$ DPBS containing 0.09\% sodium azide and $3 \%$ heat-inactivated FBS), prior to flow cytometry analysis of cell cycles. Furthermore, additional fractions of cells were allowed for 48-h growth in 60-mm cell culture plate and subjected to apoptosis analysis by flow cytometry. The cells were pelleted by centrifuging at $1000 \times \mathrm{g}$ for $5 \mathrm{~min}$ and washed by PBS for three times, before being incubated for 15 min with $5 \mu \mathrm{L}$ of Annexin V-FITC and $10 \mu \mathrm{L}$ of propidium iodide (PI) in $195 \mu \mathrm{L}$ of the binding buffer. The results were analyzed by the FlowJo 7.6.1 software (FlowJo, Ashland, OR, USA) before being presented.

\section{Statistical analysis}

Statistical significance of changes in reporter activity and other gene expression was determined using either the Student's t-test or Multiple Analysis of Variations (MANOVA). The resulting data are shown as a fold change (mean \pm S.D), each of which represents at least 3 independent experiments that were each performed triplicate.

Author contributions: S.H. performed most experiments with help of L.Q. and M.W., and collected all the relevant data, except that Y.X. did a few of experiments as shown in Figure 1. S.H. also made draft of this manuscript with most figures and supplemental information. Y.Z. designed and supervised this study, analyzed all the data, helped to prepare all figures with cartoons, wrote and revised the paper.

Acknowledgments: We are greatly thankful to Dr. Yonggang Ren (North Sichuan Medical College, Sichuan, China) for his involvement in establishing the indicated cell lines used in this study. We also thank to other members of Prof. Zhang's laboratory (at Chongqing University, China) for giving their invaluable help with this work. Notably, this study was funded by the National Natural Science Foundation of China (NSFC, with a key program 91429305 and additional two projects 81872336 and 82073079) awarded to Prof. Yiguo Zhang.

Conflicts of Interest: The authors declare no conflict of interest.

\section{References}

Baldridge, R.D., and Rapoport, T.A. (2016). Autoubiquitination of the Hrd1 Ligase Triggers Protein Retrotranslocation in ERAD. Cell 166, 394-407. 
Bugno, M., Daniel, M., Chepelev, N.L., and Willmore, W.G. (2015). Changing gears in Nrf1 research, from mechanisms of regulation to its role in disease and prevention. Biochim Biophys Acta 1849, 1260-1276.

Chen, J., Wang, M., Xiang, Y., Ru, X., Ren, Y., Liu, X., Qiu, L., and Zhang, Y. (2020). Nrf1 Is Endowed with a Dominant Tumor-Repressing Effect onto the Wnt/beta-Catenin-Dependent and Wnt/beta-Catenin-Independent Signaling Networks in the Human Liver Cancer. Oxidative medicine and cellular longevity 2020, 5138539 .

Clague, M.J., Urbe, S., and Komander, D. (2019). Breaking the chains: deubiquitylating enzyme specificity begets function. Nature reviews Molecular cell biology 20, 338-352.

Coyne, E.S., and Wing, S.S. (2016). The business of deubiquitination - location, location, location. F1000Research 5, 163.

Eletr, Z.M., and Wilkinson, K.D. (2014). Regulation of proteolysis by human deubiquitinating enzymes. Biochim Biophys Acta 1843, 114-128.

Fukagai, K., Waku, T., Chowdhury, A.M., Kubo, K., Matsumoto, M., Kato, H., Natsume, T., Tsuruta, F., Chiba, T., Taniguchi, H., et al. (2016). USP15 stabilizes the transcription factor Nrf1 in the nucleus, promoting the proteasome gene expression. Biochemical and biophysical research communications 478, 363-370.

Gegotek, A., and Skrzydlewska, E. (2015). CNC proteins in physiology and pathology. Postepy Hig Med Dosw (Online) 69, 729-743.

Hamazaki, J., and Murata, S. (2020). ER-Resident Transcription Factor Nrf1 Regulates Proteasome Expression and Beyond. International journal of molecular sciences 21, 3683.

Hassink, G.C., Zhao, B., Sompallae, R., Altun, M., Gastaldello, S., Zinin, N.V., Masucci, M.G., and Lindsten, K. (2009). The ER-resident ubiquitin-specific protease 19 participates in the UPR and rescues ERAD substrates. EMBO reports 10, 755-761.

Hayes, J.D., Dinkova-Kostova, A.T., and Tew, K.D. (2020). Oxidative Stress in Cancer. Cancer cell 38, 167-197.

Hwang, J., Walczak, C.P., Shaler, T.A., Olzmann, J.A., Zhang, L., Elias, J.E., and Kopito, R.R. (2017). Characterization of protein complexes of the endoplasmic reticulum-associated degradation E3 ubiquitin ligase Hrd1. The Journal of biological chemistry 292, 9104-9116.

Koizumi, S., Irie, T., Hirayama, S., Sakurai, Y., Yashiroda, H., Naguro, I., Ichijo, H., Hamazaki, J., and Murata, S. (2016). The aspartyl protease DDI2 activates Nrf1 to compensate for proteasome dysfunction. eLife 5, e18357. Lehrbach, N.J., Breen, P.C., and Ruvkun, G. (2019). Protein Sequence Editing of SKN-1A/Nrf1 by Peptide:N-Glycanase Controls Proteasome Gene Expression. Cell 177, 737-750

Lehrbach, N.J., and Ruvkun, G. (2016). Proteasome dysfunction triggers activation of SKN-1A/Nrf1 by the aspartic protease DDI-1. eLife 5, e17721.

Lei, C.Q., Wu, X., Zhong, X., Jiang, L., Zhong, B., and Shu, H.B. (2019). USP19 Inhibits TNF-alpha- and IL-1beta-Triggered NF-kappaB Activation by Deubiquitinating TAK1. Journal of immunology 203, 259-268.

Lynn, D.A., and Curran, S.P. (2015). The SKN-1 hunger games: May the odds be ever in your favor. Worm 4, e1078959.

Miao, R., Lu, Y., He, X., Liu, X., Chen, Z., and Wang, J. (2020). Ubiquitin-specific protease 19 blunts pathological cardiac hypertrophy via inhibition of the TAK1-dependent pathway. Journal of cellular and molecular medicine 00,1-12.

Qiu, L., Wang, M., Hu, S., Ru, X., Ren, Y., Zhang, Z., Yu, S., and Zhang, Y. (2018). Oncogenic Activation of Nrf2, Though as a Master Antioxidant Transcription Factor, Liberated by Specific Knockout of the Full-Length Nrf1alpha that Acts as a Dominant Tumor Repressor. Cancers 10, 520.

Ren, Y., Qiu, L., Lü, F., Ru, X., Li, S., Xiang, Y., Yu, S., and Zhang, Y. (2016). TALENs-directed knockout of the full-length transcription factor Nrf1a that represses malignant behaviour of human hepatocellular carcinoma 
(HepG2) cells Scientific reports 6, 23775.

Sha, Z., and Goldberg, A.L. (2014). Proteasome-mediated processing of Nrf1 is essential for coordinate induction of all proteasome subunits and p97. Current biology : CB 24, 1573-1583.

Steffen, J., Seeger, M., Koch, A., and Kruger, E. (2010). Proteasomal degradation is transcriptionally controlled by TCF11 via an ERAD-dependent feedback loop. Molecular cell 40, 147-158.

Sykiotis, G.P., and Bohmann, D. (2010). Stress-activated cap'n'collar transcription factors in aging and human disease. Science signaling 3, re3.

Taniguchi, H., Okamuro, S., Koji, M., Waku, T., Kubo, K., Hatanaka, A., Sun, Y., Chowdhury, A.M., Fukamizu, A., and Kobayashi, A. (2017). Possible roles of the transcription factor Nrf1 (NFE2L1) in neural homeostasis by regulating the gene expression of deubiquitinating enzymes. Biochemical and biophysical research communications 484, 176-183.

Tsuchiya, Y., Morita, T., Kim, M., lemura, S., Natsume, T., Yamamoto, M., and Kobayashi, A. (2011). Dual regulation of the transcriptional activity of $\mathrm{Nrf1}$ by beta-TrCP- and Hrd1-dependent degradation mechanisms. Molecular and cellular biology 31, 4500-4512.

Ventii, K.H., and Wilkinson, K.D. (2008). Protein partners of deubiquitinating enzymes. The Biochemical journal 414, 161-175.

Villeneuve, N.F., Tian, W., Wu, T., Sun, Z., Lau, A., Chapman, E., Fang, D., and Zhang, D.D. (2013). USP15 negatively regulates Nrf2 through deubiquitination of Keap1. Molecular cell 51, 68-79.

Widenmaier, S.B., Snyder, N.A., Nguyen, T.B., Arduini, A., Lee, G.Y., Arruda, A.P., Saksi, J., Bartelt, A., and Hotamisligil, G.S. (2017). NRF1 Is an ER Membrane Sensor that Is Central to Cholesterol Homeostasis. Cell 171, 1094-1109

Xiang, Y., Halin, J., Fan, Z., Hu, S., Wang, M., Qiu, L., Zhang, Z., Mattjus, P., and Zhang, Y. (2018a). Topovectorial mechanisms control the juxtamembrane proteolytic processing of $\mathrm{Nrf} 1$ to remove its $\mathrm{N}$-terminal polypeptides during maturation of the CNC-bZIP factor. Toxicology and applied pharmacology 360, 160-184.

Xiang, Y., Wang, M., Hu, S., Qiu, L., Yang, F., Zhang, Z., Yu, S., Pi, J., and Zhang, Y. (2018b). Mechanisms controlling the multistage post-translational processing of endogenous Nrf1alpha/TCF11 proteins to yield distinct isoforms within the coupled positive and negative feedback circuits. Toxicology and applied pharmacology 360, 212-235.

Xu, Y., Cui, L., Dibello, A., Wang, L., Lee, J., Saidi, L., Lee, J.G., and Ye, Y. (2018). DNAJC5 facilitates USP19-dependent unconventional secretion of misfolded cytosolic proteins. Cell discovery 4, 11.

Yamamoto, M., Kensler, T.W., and Motohashi, H. (2018). The KEAP1-NRF2 System: a Thiol-Based Sensor-Effector Apparatus for Maintaining Redox Homeostasis. Physiological reviews 98, 1169-1203.

Zhang, Y., Crouch, D.H., Yamamoto, M., and Hayes, J.D. (2006). Negative regulation of the Nrf1 transcription factor by its $\mathrm{N}$-terminal domain is independent of Keap1: Nrf1, but not Nrf2, is targeted to the endoplasmic reticulum. The Biochemical journal 399, 373-385.

Zhang, Y., Li, S., Xiang, Y., Qiu, L., Zhao, H., and Hayes, J.D. (2015). The selective post-translational processing of transcription factor Nrf1 yields distinct isoforms that dictate its ability to differentially regulate gene expression. Scientific reports 5, 12983.

Zhang, Y., Lucocq, J.M., and Hayes, J.D. (2009). The Nrf1 CNC/bZIP protein is a nuclear envelope-bound transcription factor that is activated by t-butyl hydroquinone but not by endoplasmic reticulum stressors. The Biochemical journal 418, 293-310.

Zhang, Y., Lucocq, J.M., Yamamoto, M., and Hayes, J.D. (2007). The NHB1 (N-terminal homology box 1) sequence in transcription factor Nrf1 is required to anchor it to the endoplasmic reticulum and also to enable its asparagine-glycosylation. The Biochemical journal 408, 161-172. 
Zhang, Y., Ren, Y., Li, S., and Hayes, J.D. (2014). Transcription factor Nrf1 is topologically repartitioned across membranes to enable target gene transactivation through its acidic glucose-responsive domains. PloS one 9 , e93458.

Zhang, Y., and Xiang, Y. (2016). Molecular and cellular basis for the unique functioning of Nrf1, an indispensable transcription factor for maintaining cell homoeostasis and organ integrity. The Biochemical journal 473, 961-1000.

Zhu, Y.P., Wang, M., Xiang, Y., Qiu, L., Hu, S., Zhang, Z., Mattjus, P., Zhu, X., and Zhang, Y. (2018). Nach Is a Novel Subgroup at an Early Evolutionary Stage of the CNC-bZIP Subfamily Transcription Factors from the Marine Bacteria to Humans. International journal of molecular sciences 19, 1-26.

Zhu, Y.P., Zheng, Z., Hu, S., Ru, X., Fan, Z., Qiu, L., and Zhang, Y. (2019). Unification of Opposites between Two Antioxidant Transcription Factors Nrf1 and Nrf2 in Mediating Distinct Cellular Responses to the Endoplasmic Reticulum Stressor Tunicamycin. Antioxidants 9, 4. 